\title{
Spatiotemporal distribution of microbial communities in a coastal, sandy aquifer system (Doñana, SW Spain)
}

\author{
SERGIO VELASCO, ${ }^{1,2}$ MARÍA DEL CARMEN GUERRERO,${ }^{1}$ CARLOS MONTES ${ }^{1}$ AND \\ ANA ISABEL LÓPEZ-ARCHILLA ${ }^{1}$ \\ ${ }^{1}$ Departamento de Ecología, Facultad de Ciencias, Universidad Autónoma de Madrid, Cantoblanco, 28049 Madrid, Spain \\ ${ }^{2}$ Dirección de Estudios, Agua y Medio Abiente, Área de Medio Ambiente Hidrico, Centro de Estudios Hidrográficos, CEDEX, \\ Ministerio de Fomento, 28005 Madrid, Spain
}

\begin{abstract}
The aquifer system of Doñana (SW Spain) represents the most important freshwater source in the Doñana Natural Area. Its spatiotemporal dynamics favours the hydrological connection between surface and subsurface ecosystems, and promotes matter fluxes among the different terrestrial and aquatic systems present here. This aquifer has been intensively studied from a hydrogeological point of view but little is known from an ecological perspective. In order to understand the ecological roles played by microbial communities in this system, we conducted a long-term seasonal study of bacterial abundance, cell biomass, bacterial biomass and functional activities over a 2-year period. Bacterial abundance ranged between $2.11 \pm 1.79 \times 10^{5}$ and $8.58 \pm 6.99 \times 10^{7}$ bact $\mathrm{mL}^{-1}$ groundwater, average cell biomass was estimated to be $77.01 \pm 31.56 \mathrm{fgC}$ and bacterial biomass varied between $8.99 \pm 4.10 \times 10^{-2}$ and $5.65 \pm 0.70 \mu \mathrm{gC} \mathrm{mL}-1$. Iron-related bacteria showed the highest activities among the functional groups studied. Moreover, among the variables that usually control spatial distributions of microbial communities in aquifer systems, depth did not have a relevant effect on this aquifer, at least in the range of depths studied, but grain size, probably due to its direct effects on hydrogeological parameters, such as permeability or porosity, appeared to exert moderate control, principally in terms of bacterial abundance. Finally, significant seasonal differences in the means of these microbiological variables were also observed; temperature seems to be the main factor controlling the temporal distribution of microbial communities in this aquifer system.
\end{abstract}

Received 00 xxxx 2008; accepted 00 xxxx 2008

Corresponding author: Sergio Velasco, Tel.: xxxxxxxxx; fax: xxxxxxxxx; e-mail: sergio.velasco@uam.es.

\section{INTRODUCTION}

The paradigm in the study of aquifer systems has changed over the last few decades from a pure hydrogeological point of view to a more ecological one (Danielopol, 1989; Baker et al., 2000). As a consequence, aquifer systems are currently considered to constitute a heterogeneous assemblage of discrete macro- and microscale habitats, providing a variety of living conditions (Goldscheider et al., 2006).

From an ecological standpoint, aquifer systems should be regarded as open systems interchanging materials and energy with other aquatic and terrestrial systems located in the vicinity (Danielopol, 1989; Chapelle, 2000b; Danielopol et al., 2003). The recognition of interactions occurring within and among surrounding environments over a range of scales will allow for a better understanding of the ecological roles played by microbial communities in aquifer systems (Brockman \& Murray, 1997; Bennett et al., 2000; Musslewhite et al., 2003; Hancock et al., 2005). However, the identification of such interactions requires long-term spatiotemporal studies, very rare to date. Multidisciplinary studies would be of use in this identification only if all the approximations share similar spatiotemporal scales (Brockman \& Murray, 1997; Musslewhite et al., 2003).

Microbial ecology studies of sedimentary, relatively shallow aquifer systems are relatively abundant (Marxsen, 1988; Alfreider et al., 1997; Martino et al., 1998; Griebler et al., 2002; Velasco et al., 2008; in press), but very few exist with large spatial and temporal scales. Some of these studies have shown aquifers as more stable ecosystems, with less spatiotemporal variation in their ecological processes, than other aquatic systems. The main goal of the present study, conducted in an area of approximately 
$100 \mathrm{~km}^{2}$ over a 2 -year period, is to enhance our knowledge of 2 the microbial communities existing in the aquifer system of Doñana, an ecosystem in which there have been only a few studies based on a biological or an ecological approach. This aquifer is becoming to be considered as part of a great ecosystem, called the bydroecosystem, made up of several aquatic and terrestrial systems, and located in the Doñana Natural Area (Montes et al., 1998; Manzano \& Custodio, 2007; Manzano et al., 2007). All these ecosystems in the Doñana Natural Area make it unique in many senses: it is a major stepping-stone in the migration route of birds moving between Europe and Africa, it is home to the most endangered mammals in the world, as well as many endemic, threatened or ecologically interesting species, and it contains what is possibly Europe's most significant wetland. Consequently, there has been an increasing number of interdisciplinary studies in the last few years, and groups of limnologists, microbiologists, hydrogeologists, economists and sociologists are working together in order to obtain a general overview of the ecological functioning of Doñana's hydroecosystem (Manzano et al., 2007).

Bearing in mind that the most important freshwater source in the Doñana Natural Area is the groundwater, and that the aquifer controls the hydrological regime of all other ecosystems located in this area, it is important to understand the role played by microbial communities present in the aquifer system. To this end, the first step is to define these microbial communities in terms of bacterial abundance and cell biomass (Murphy \& Schramke, 1998), because this information will enable the potential activity of the population to be identified (Bratbak, 1993). Knowledge of factors, such as depth, grain size or temperature, controlling spatial and temporal patterns in bacterial abundances, cell biomasses and microbial activities are also important to characterize the distribution and dynamics of these communities. The results of this paper complement and consolidate others resulting from a parallel, intensive study, in which microbial communities present in the groundwater located in the surroundings of four very productive shallow lakes were studied by sampling 13 piezometers, different from those employed in this paper (Velasco et al., 2008; in press).

\section{MATERIALS AND METHODS}

\section{Site description}

The Doñana aquifer system is included in the Doñana Natural Area and located in the SW Atlantic coast of Spain. The aquifer has a surface area of around $3000 \mathrm{~km}^{2}$ and hosts the Guadalquivir river marshes and Doñana National and Natural Parks, two protected natural areas of international relevance 50 comprising approximately $1100 \mathrm{~km}^{2}$ (Fig. 1). They were 51 declared Biosphere Reserves in 1981, RAMSAR Sites in 1982 52 and Natural World Heritage Sites in 1994. The climate is 53 Mediterranean subhumid with Atlantic influence: dry summers 54 and wet winters. Mean rainfall, mainly concentrated from
October to March, is $500-600 \mathrm{~mm}$, but has a high interannual variability. Mean yearly temperature is around $17^{\circ} \mathrm{C}$ near the coast and $18{ }^{\circ} \mathrm{C}$ in the centre of the Natural Area (Manzano et al., 2007).

The aquifer system consists of detrital, unconsolidated Plio-Quaternary sediments overlapping impervious Miocene marine marls. The Pliocene materials are impermeable marls, silts and sandy silts. The Quaternary materials consist of deltaic and alluvial silts, sands and gravels to the north, and on littoral, alluvial and aeolian sands to the west. They mainly comprise amorphous silica grains. Carbonates may be present either as detrital grains or as shell remains, except in the upper part of the aeolian sand layers of the western sector (Manzano et al., 2007). The Quaternary layers thicken from $\mathrm{N}$ to $S$ and from $\mathrm{W}$ to SE. To the SE, the coarse sediments are covered by a thick $(50-80 \mathrm{~m})$ sequence of estuarine and marshy clays. The aquifer system varies in thickness, ranging from $20 \mathrm{~m}$ inland to over $150 \mathrm{~m}$ at the coast line. At regional scale, the aquifer system presents two lithological domains: a sandy one to the $\mathrm{N}$ and $\mathrm{W}$ of the marshes, which extensive areas of aeolian sands, which roughly behaves as an unconfined aquifer, and a clayey one in the marsh area, under which a large confined aquifer is found (Manzano et al., 2007). In the aeolian sands, there are two different lithological subdomains (called upper and lower units) that allow for the presence of two different hydrodynamic units. The thick and fine to medium sand deposits of the upper unit conform with a relatively homogeneous phreatic aquifer that contains the water table and overlies a lower, less homogeneous and semiconfined aquifer composed of coarse sands and gravels (Trick \& Custodio, 2004). The hydraulic transmissivity of the lower, thinner aquifer is higher than that of the upper one. Between the upper and the lower units there is an intermediate layer of grey clays and fine to medium clayed sands containing iron oxide minerals. Recharge occurs via direct rain infiltration in the aeolian sands (Trick \& Custodio, 2004). Groundwater mainly flows eastward from aeolian sands in the west to the ecotone and the marsh in the east (Manzano et al., 2007) (Fig. 1). Natural discharge takes place to the ocean, rivers and ravines, to many small phreatic shallow lakes situated above the aeolian mantles (Coleto, 2003), and through phreatic evapotranspiration (Manzano \& Custodio, 2007). In general, there are vertical, descending flows in recharge areas and vertical, ascending flows in discharge areas and groundwater extraction points (Trick \& Custodio, 2004).

\section{Sampling procedure: physical and chemical variables}

Groundwater samples were collected seasonally (winter 2003 to winter 2005) from 17 piezometers located in an area encompassing approximately $100 \mathrm{~km}^{2}$ (Fig. 1). Except well piso (installed and monitored by the Universidad Autónoma de Madrid, UAM), all boreholes were installed and are monitored by the Spanish Geological Survey and the Guadalquivir River Basin Authority. Screen depth of piezometers ranges from 
1
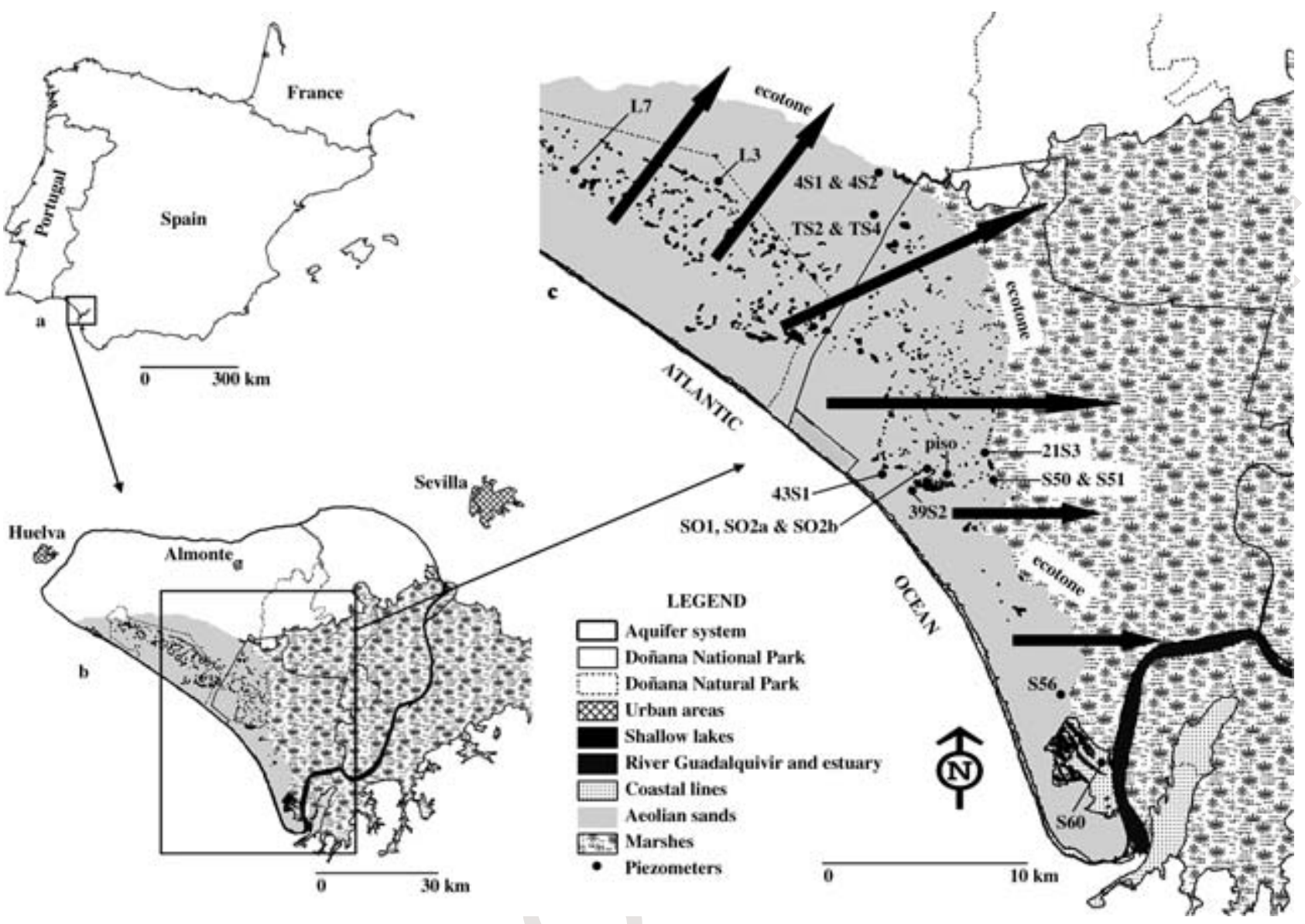

Fig. 1 Geographical location of the Doñana Natural Area on the south-west coast of the Iberian Peninsula (A). The figure also shows the boundary of the aquifer system of Doñana, the boundaries of the Doñana National and Natural Parks, as well as the most important ecosystems in the Doñana Natural Area (B). Both shallow lakes and the 17 studied piezometers are also shown. Arrows indicate the general direction of the regional groundwater flow from east (aeolian sands) to west (marshes) (C).

3.00 to 80.00 meters below land surface (mbls) and all are located in the aeolian sands (both in the upper and in the lower unit) (Table 1).

Groundwater samples were collected following chemical (Dunlap et al., 1977) and microbiological (Fredrickson \& Phelps, 1997) standard procedures. A special pneumatic pump was employed to avoid mixing processes between groundwater and retained water in the piezometer (Danielopol \& Niederreiter, 1987). Groundwater was pumped from each well until temperature, dissolved oxygen (DO), $\mathrm{pH}$ and electric conductivity readings stabilized (Manzano et al., 2007). All physical parameters were measured with a WTW 340i handheld multiparameter device. Chemical parameters (alkalinity, ammonium, nitrate, nitrite, soluble reactive phosphorus (SRP) and total phosphorus (TP)) were estimated by means of standard methods during the 10 following days (APHA et al., 1987); alkalinity was analysed during the same sampling day (APHA et al., 1987). Ferrous and ferric iron concentrations, as well as total iron, were determined by the ferrozine colorimetric method (Viollier et al., 2000).

\section{Microbiological variables}

Groundwater samples for microbiological variables were collected from each well in triplicate, stored in $100 \mathrm{~mL}$ polyurethane bottles (prewashed in $5 \% \mathrm{HCl}$ and distilled water), and fixed with formaldehyde $(2 \% \mathrm{v} / \mathrm{v}$ final concentration). Samples were stored in the dark at $4{ }^{\circ} \mathrm{C}$ until the filtration process. Bacterial abundance was determined by epifluorescence microscopy after staining with DAPI (4',6-diamidino-2-phenylindoledihydrochloride) $1 \mu \mathrm{g} \mathrm{mL}^{-1}$ final concentration (Fry, 1990). The solution was then filtered through a $0.2-\mu \mathrm{m}$ pore size GTBP $25 \mathrm{~mm} \emptyset$ Millipore filter (Millipore Inc., Billerica, MA, USA). Filters were transferred to a labelled microscope slide and kept frozen until samples could be counted (Bölter $e t$ al., 2002). Three different filters were prepared for each sampled well per season, and 20 different fields were viewed and counted for each filter (Kircl $\equiv$ et al., 1982) with an Olympus IX50 inverted microscope. The results of the counts for bacterial abundance are presented as bact $\mathrm{mL}^{-1}$ groundwater. Length and width measurements of bacterial cells under a magnification 
Table 1 Geographical, lithological and hydrogeological features of the selected piezometers

\begin{tabular}{|c|c|c|c|c|c|c|c|c|c|}
\hline Piezometer & $\begin{array}{l}\text { UTM } \\
\times(29)\end{array}$ & $\begin{array}{l}\text { UTM } \\
\text { Y (29) }\end{array}$ & $\begin{array}{l}\text { Altitude } \\
\text { (masl) }\end{array}$ & $\begin{array}{l}\text { Screen } \\
\text { Depth (mbls) }\end{array}$ & $\begin{array}{l}\text { Hydrogeological } \\
\text { Unit* }\end{array}$ & $\begin{array}{l}\text { Screen } \\
\text { Lithology }\end{array}$ & $\begin{array}{l}\text { Transmissivity } \\
\left(\mathrm{m}^{2} \text { day }^{-1}\right)^{\dagger}\end{array}$ & $\begin{array}{l}\text { Permeability } \\
\left(\mathrm{m} \mathrm{day}^{-1}\right)^{\dagger}\end{array}$ & Contamination $^{\dagger}$ \\
\hline Piso & 724598 & 4095905 & 5.50 & $2.00-2.25$ & U & Fine to medium sands & & & \\
\hline S51 & 727730 & 4097026 & 2.00 & $3.00-7.00$ & $U$ & Fine sands & 1.13 & 0.28 & \\
\hline $21 \mathrm{S3}$ & 727969 & 4101313 & 4.00 & $5.40-8.20$ & $U$ & Fine sands & & & \\
\hline $4 \mathrm{~S} 1$ & 722485 & 4111933 & 4.38 & $8.00-10.00$ & $U$ & Fine to medium sands & & & Nitrate \\
\hline L3 & 711081 & 4111414 & 43.00 & $8.00-10.00$ & $U$ & Fine to medium sands & 0.20 & 0.10 & \\
\hline L7 & 700031 & 4113890 & 69.00 & $8.00-10.00$ & $U$ & Coarse sands & & & Nitrate \\
\hline 3952 & 723848 & 4095698 & 5.70 & $8.50-11.40$ & U & Fine to medium sands & & & \\
\hline TS4 & 719101 & 4112389 & 16.30 & $10.00-11.00$ & $U$ & Fine to medium sands & 3.00 & 3.00 & Nitrate \\
\hline 4351 & 722031 & 4096458 & 11.30 & $11.50-14.30$ & $U$ & Fine to medium sands & 0.13 & 0.05 & \\
\hline S60 & 734318 & 4080575 & 3.00 & $16.00-17.00$ & $U$ & Fine sands & & & \\
\hline TS2 & 719101 & 4112389 & 16.30 & $18.00-19.00$ & INT & Clays & 0.50 & 0.50 & Nitrate \\
\hline $\mathrm{SO} 2 \mathrm{a}$ & 724189 & 4096032 & 6.00 & $44.00-46.00$ & $U$ & Fine to medium sands & & & \\
\hline $4 \mathrm{~S} 2$ & 722485 & 4111933 & 4.57 & $36.50-43.50$ & $\mathrm{~L}$ & Clays and fine sands & & & Nitrate \\
\hline $\mathrm{SO} 2 \mathrm{~b}$ & 724189 & 4096032 & 6.00 & $44.00-46.00$ & $U$ & Fine to medium sands & & & \\
\hline S50 & 727730 & 4097026 & 3.00 & $52.00-60.00$ & $\mathrm{~L}$ & Gravels and coarse sands & & & \\
\hline S56 & 733010 & 4087500 & 2.00 & $74.00-80.00$ & L & Gravels and coarse sands & & & \\
\hline SO1 & 724188 & 4096038 & 6.00 & $67.00-72.00$ & $U$ & Fine to medium sands & & & \\
\hline
\end{tabular}

*Location of $\bar{\equiv}$.lls in the hydrogeological units (U, upper; L, lower; INT, intermediate).

'Data from Trick 1998.

masl, metres above sea level; mbls, metres below land surface.

of $\times 1000$ were used to determine the cell biovolume; 60 cells were measured in each filter and were classified as cocci, small and large rods, and filamentous bacteria. Biovolumes of bacterial cells were calculated assuming that the shapes of the cells were either perfect spheres or cylinders with hemispheres on both sides (Fry, 1990). Linear dimensions were converted to volumetric units using geometric formulas (Bölter et al., 2002). Cell biomass was calculated from the cell biovolume using a conversion factor based on the allometric model with the formula $\mathrm{C}=120 \times \mathrm{V}^{0.7}$, where $\mathrm{C}=$ cell biomass in $\mathrm{fgC}$ and $\mathrm{V}=$ cell biovolume in $\mu \mathrm{m}^{3}$ (Psenner, 1993). Cell biomass is presented as $\mathrm{fgC}$. The product of the bacterial abundance and the cell biomass is the bacterial or population biomass, which is shown as $\mu \mathrm{gC} \mathrm{mL} \mathrm{m}^{-1}$ groundwater.

Evidence of microbial activity for some functional groups of bacteria (nitrifying $(\mathrm{NB})$, denitrifying $(\mathrm{DNB})$, iron related (IRB), and sulphate-reducing bacteria (SRB)) was provided by commercial biological activity reaction tests $\left(\mathrm{BART}^{\mathrm{TM}}\right)$ (Cullimore, 1993). A BART ${ }^{\mathrm{TM}}$ test is a simple and effective method for detecting the presence and the relative potential activity of a specific functional group of microorganisms. Briefly, three $10 \mathrm{~mL}$ replicates of groundwater were incubated during 10 days at room temperature for each functional group. The first day following incubation in which the activity was detected, was selected as the start of activity by a specific functional group. The more time required for the detection of a specific activity, the less activity the functional group showed. Assays were carried out during winters and summers (years 2003 and 2004).

\section{Hydrogeological variables}

Transmissivity and permeability values for some piezometers were previously measured (Trick \& Custodio, 2004). Rainfall data were obtained with permission (Spanish Meteorological Service, INM) from a sampling weather station located in Doñana National Park.

\section{Statistical analyses}

Differences in the means of bacterial abundance, cell biomass and bacterial biomass among wells were tested, for each sampling campaign, using a one-way MANova test. Temporal differences in the means of the same microbiological variables for each well sampled more than three times during the study period were also tested by means of a one-way MANOva test. Two different MANOVAs had to be performed to test the effect of one out of the two different factors (wells or seasons) because the data matrix was not regular. In other words, not all the piezometers were sampled during the same sampling campaigns, or at least the same three sampling campaigns. However, all piezometers were taken into account for statistical purposes when spatial differences were searching. Univariate F-tests were also performed after MANOva testing. Tukey's honestly significant difference test (HSD test) was employed, as an a posteriori method, to compare pairs of means after MANOva testing. Differences in the means for microbial activities of functional groups were tested, for each sampling campaign, using a one-way ANOVA test. Temporal differences in the means 
of microbial activities during both years for each functional group were tested using a one-way ANOVA test. Tukey's HSD test was employed, as an a posteriori method, to compare pairs of means after ANOVA testing. Homogeneity of variances was tested with the Levene test (Zar, 1998). Relationships among measured variables were explored using Pearson product moment correlations or Spearman rank order correlations, depending on whether the dataset in question was parametric or nonparametric in nature. Normality was examined using the Kolmogorov-Smirnov test, and variables were transformed when necessary and when possible. Relationships between physicochemical (temperature, $\mathrm{DO}, \mathrm{pH}$, electric conductivity, ammonium, nitrate, SRP, TP, ferric iron, ferrous iron, total iron and alkalinity) and microbiological (bacterial abundance, cell biomass and bacterial biomass) variables were explored throughout a canonical correlation analysis; Bartlett's $\chi^{2}$ test was used for testing the significance of the correlations between any pairs of the canonical variates (Quinn \& Keough, 2002). A principal component analysis (PCA), with seasons as the grouping variable, was performed as a multivariate ordination method to compare different seasons and to detect temporal patterns. Data were standardized for PCA (Legendre \& Legendre, 1998). A Pvalue of 0.05 was set as the significant threshold for statistical analyses. All statistical analyses were performed with Statistica 6.0 for Windows (Statsoft, Inc., Tulsa, OK, USA).

\section{RESULTS}

\section{Physicochemical variables}

Groundwater temperature showed mean values of approximately $19{ }^{\circ} \mathrm{C}$; these values increased throughout the year during both years (Table 2). Dissolved oxygen exhibited mean values of $2 \mathrm{mg} \mathrm{L}^{-1}$, although with a different temporal pattern, with higher winter and spring values than those for summer or autumn. Therefore, temperature and DO negatively correlated $(r=-0.341, P=0.004, n=71)$. The $\mathrm{pH}$ was very constant and ranged from 6 to 7 in most of the wells (Table 2). Electric conductivity (EC) values were measured around 300-400 $\mu \mathrm{S}$ $\mathrm{cm}^{-1}$; however, in some piezometers located in the ecotone, close to the marsh (Fig. 1), conductivities were higher.

Among the inorganic nitrogen forms, nitrate concentrations $\left(\mathrm{mg} \mathrm{L}^{-1} \mathrm{~N}-\mathrm{NO}_{3}^{-}\right)$were higher than ammonium $\left(\mathrm{mg} \mathrm{L}^{-1}\right.$ $\mathrm{N}-\mathrm{NH}_{4}^{+}$) or nitrite concentrations $\left(\mathrm{mg} \mathrm{L}^{-1} \mathrm{~N}-\mathrm{NO}_{2}^{-}\right.$; data not shown) in most of the boreholes (Table 3 ). In some wells, nitrate concentrations were very high (Table 3 ). Soluble

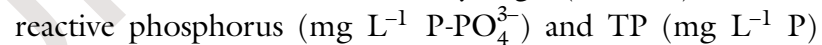
showed seasonal concentrations of approximately $0.05 \mathrm{mg} \mathrm{L}^{-1}$ and $0.08 \mathrm{mg} \mathrm{L}^{-1}$, respectively (Table 3 ). Ferric iron usually displayed higher concentrations than ferrous iron. Total iron concentration ranged from 1 to $2 \mathrm{mg} \mathrm{L}^{-1}$ in most of the wells, and reached maximum values of approximately $20 \mathrm{mg} \mathrm{L}^{-1}$ (Table 3 ). Total alkalinity values were around 1 meq $\mathrm{L}^{-1}$ (Table 2).

\section{Microbiological variables}

Only prokaryotic cells were observed in microscope counts, with dominance of rod-shaped bacteria. No differences among cell morphologies were observed in samples from different depths or from different sites. Average bacterial abundance was $1.70 \times 10^{7} \pm 1.99 \times 10^{6}$ bact $\mathrm{mL}^{-1}$ groundwater. Significant differences for bacterial abundance among piezometers were observed during the first three sampling campaigns in $2003(P \leq 0.039)$. Seasonal differences for bacterial abundance in wells sampled more than three times were observed $(P \leq 0.047)$ except in boreholes $39 \mathrm{~S} 2,43 \mathrm{~S} 1, \mathrm{SO} 2 \mathrm{a}$ and SO2b $(P \geq 0.156)$ (Fig. 2A,B). Bacterial abundances were statistically higher during summer or autumn 2003 than during winter or spring 2003 ( $P \leq 0.042$ ), except for wells $43 \mathrm{~S} 1$, SO2a, SO2b and SOl $(P \geq 0.143)$. During 2004, no statistical differences were observed among seasons $(P \geq 0.256)$. This variable positively correlated with temperature $(r=0.386, P=0.001$, $n=71)$, nitrate $(r=0.430, P=0.000, n=70)$ and cell biomass $(r=0.335, P=0.004, r=71)$, whereas it negatively correlated with DO $(r=-0.237, P=0.047, n=71)$, ferrous iron $(r=-0.316, P=0.034, n=41)$ and total iron $(r=$ $-0.265, P=0.036, n=63$ ). Bacterial abundances in wells S51, $\mathrm{SO} 2 \mathrm{a}, \mathrm{SO} 2 \mathrm{~b}$ and SO1 negatively correlated with rainfall from 2 months prior to sampling $(P \leq 0.046)$. No correlations among bacterial abundance, screen depth, permeability and transmissivity were found, except during spring 2003, when bacterial abundance and depth positively correlated $(r=0.890$, $P=0.009, n=8)$.

Mean cell biomass was determined to be $77 \pm 32 \mathrm{fgC}$ (Fig. 2C,D). Cell biomass showed significant differences among piezometers during all seasons $(P \leq 0.048)$, except in winter $2005(P \geq 0.109)$. Significant seasonal differences for cell biomass were observed in piezometers sampled more than three times during the study period $(P=0.000)$, except in well TS4 $(P=0.204)$. A significant increase in cell biomass during 2003 was observed toward the warmer seasons in all piezometers $(P \leq 0.004)$, except in wells TS4 and SO2a $(P \geq 0.654)$. A significant, although less evident, increase in cell biomass throughout 2004 was observed in wells S51, 4S1, SO2a, $\mathrm{SO} 2 \mathrm{~b}$ and $\mathrm{SO} 1$, with statistical differences between winter or spring and summer or autumn $(P \leq 0.023)$. Cell biomass positively correlated with temperature $(r=0.487, P=0.000$, $n=71)$ and negatively with DO $(r=-0.242, P=0.037$, $n=71)$. In wells S51 and SOl cell biomass negatively correlated with rainfall from 2 months prior to sampling $(P \leq 0.028)$. No significant correlations were found among cell biomass, depth, permeability and transmissivity in any season.

Average bacterial biomass was $1.40 \pm 0.57 \mu \mathrm{gC} \mathrm{mL} \mathrm{mL}^{-1}$. Differences in the means of bacterial biomass among piezometers were statistically significant in all seasons $(P=0.000)$, but more evident during 2003 than during 2004. Seasonal differences were observed in all piezometers sampled more than three times throughout the study period $(P=0.000)$ (Fig. $2 \mathrm{E}, \mathrm{F})$. 
Table 2 Physical variables and alkalinity seasonally measured in the different wells (mean \pm standard deviation) (T, temperature; DO, dissolved oxygen; EC, electric conductivity; win, winter; spr, spring; sum, summer; aut, autumn)

\begin{tabular}{|c|c|c|c|c|c|c|}
\hline Piezometer & Season & $\mathrm{T}\left({ }^{\circ} \mathrm{C}\right)$ & $\begin{array}{l}\mathrm{DO} \\
\left(\mathrm{mg} \mathrm{L}^{-1}\right)\end{array}$ & $\mathrm{pH}$ & 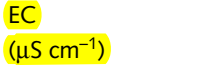 & $\begin{array}{l}\text { Alkalinity } \\
\text { (meq L-1) }\end{array}$ \\
\hline \multirow[t]{5}{*}{ Piso } & sum-03 & $19.90 \pm 0.26$ & $2.30 \pm 0.10$ & $6.76 \pm 0.24$ & $202.20 \pm 0.26$ & $0.61 \pm 0.12$ \\
\hline & aut-03 & $20.63 \pm 0.35$ & $1.83 \pm 0.06$ & $7.16 \pm 0.15$ & $172.52 \pm 1.41$ & $0.50 \pm 0.01$ \\
\hline & sum-04 & $22.53 \pm 0.15$ & $2.57 \pm 0.05$ & $6.39 \pm 0.01$ & $263.67 \pm 2.08$ & $0.57 \pm 0.06$ \\
\hline & aut-04 & $21.30 \pm 0.10$ & $2.16 \pm 0.00$ & $6.51 \pm 0.02$ & $210.67 \pm 3.21$ & $0.53 \pm 0.06$ \\
\hline & win-05 & $15.90 \pm 0.20$ & $1.97 \pm 0.01$ & $6.78 \pm 0.07$ & $235.67 \pm 7.37$ & $0.27 \pm 0.11$ \\
\hline \multirow[t]{6}{*}{ S51 } & win-03 & 14.90 & 5.48 & 8.30 & 366.00 & \\
\hline & spr-03 & $19.70 \pm 0.28$ & $1.90 \pm 0.49$ & $7.89 \pm 0.01$ & $303.67 \pm 0.09$ & $1.92 \pm 0.01$ \\
\hline & aut-03 & $21.07 \pm 0.41$ & $1.07 \pm 0.04$ & $8.14 \pm 0.11$ & $276.00 \pm 2.64$ & $1.95 \pm 0.06$ \\
\hline & spr-04 & $18.03 \pm 0.15$ & $1.97 \pm 0.07$ & $8.15 \pm 0.13$ & $246.37 \pm 0.57$ & $1.43 \pm 0.08$ \\
\hline & aut-04 & $20.17 \pm 0.05$ & $1.26 \pm 0.00$ & $8.21 \pm 0.01$ & $339.67 \pm 17.95$ & $1.60 \pm 0.00$ \\
\hline & win-05 & $18.70 \pm 0.10$ & $1.86 \pm 0.02$ & $8.04 \pm 0.00$ & $303.67 \pm 3.06$ & $1.27 \pm 0.11$ \\
\hline \multirow[t]{4}{*}{$21 S 3$} & win-03 & 17.40 & 1.12 & 7.33 & 184.10 & \\
\hline & spr-03 & $18.65 \pm 0.63$ & $1.49 \pm 0.48$ & $7.15 \pm 0.21$ & $177.95 \pm 7.70$ & $0.87 \pm 0.01$ \\
\hline & sum-03 & $22.23 \pm 0.05$ & $1.40 \pm 0.17$ & $7.67 \pm 0.01$ & $169.27 \pm 1.25$ & $1.22 \pm 0.10$ \\
\hline & aut-03 & $21.97 \pm 0.40$ & $0.97 \pm 0.15$ & $7.50 \pm 0.12$ & $168.30 \pm 0.98$ & $0.97 \pm 0.08$ \\
\hline \multirow[t]{7}{*}{$4 \mathrm{~S} 1$} & spr-03 & $19.30 \pm 0.04$ & $1.75 \pm 0.07$ & $7.30 \pm 0.16$ & $290.50 \pm 0.26$ & $0.45 \pm 0.00$ \\
\hline & sum-03 & $20.07 \pm 0.05$ & $0.77 \pm 0.15$ & $7.12 \pm 0.14$ & $326.70 \pm 8.46$ & $2.31 \pm 0.28$ \\
\hline & aut-03 & $20.03 \pm 0.40$ & $0.47 \pm 0.12$ & $7.65 \pm 0.41$ & $313.00 \pm 6.92$ & $2.05 \pm 0.05$ \\
\hline & aut-04 & $18.17 \pm 0.15$ & $0.92 \pm 0.02$ & $7.83 \pm 0.00$ & $250.00 \pm 1.02$ & $2.08 \pm 0.08$ \\
\hline & sum-04 & $20.50 \pm 0.19$ & $1.26 \pm 0.04$ & $7.08 \pm 0.01$ & $300.00 \pm 2.64$ & $1.40 \pm 0.20$ \\
\hline & aut-04 & $18.83 \pm 0.37$ & $2.57 \pm 0.00$ & $7.31 \pm 0.00$ & $299.33 \pm 1.15$ & $1.60 \pm 0.00$ \\
\hline & win-05 & $18.30 \pm 0.40$ & $2.59 \pm 0.01$ & $7.09 \pm 0.01$ & $264.00 \pm 0.00$ & $1.07 \pm 0.23$ \\
\hline \multirow[t]{2}{*}{ L3 } & sum-03 & $19.45 \pm 0.07$ & $3.55 \pm 0.04$ & $6.08 \pm 0.07$ & $74.42 \pm 3.79$ & $0.40 \pm 0.00$ \\
\hline & spr-04 & $19.33 \pm 0.05$ & $4.36 \pm 0.07$ & $5.87 \pm 0.01$ & $66.33 \pm 1.52$ & $0.55 \pm 0.02$ \\
\hline \multirow[t]{4}{*}{ L7 } & win-03 & 17.30 & 6.20 & 6.03 & 796.00 & \\
\hline & sum-03 & $19.36 \pm 0.20$ & $2.40 \pm 0.09$ & $6.54 \pm 0.07$ & $760.50 \pm 6.50$ & $1.46 \pm 0.24$ \\
\hline & sum-04 & $22.10 \pm 0.09$ & $1.49 \pm 0.00$ & $6.90 \pm 0.03$ & $463.33 \pm 1.52$ & $1.27 \pm 0.12$ \\
\hline & aut-04 & $19.43 \pm 0.11$ & $1.56 \pm 0.00$ & $6.51 \pm 0.00$ & $474.33 \pm 0.57$ & $1.30 \pm 0.10$ \\
\hline \multirow[t]{5}{*}{$39 S 2$} & win-04 & $17.33 \pm 0.15$ & $2.74 \pm 0.10$ & $6.62 \pm 0.01$ & $136.67 \pm 4.72$ & $0.72 \pm 0.17$ \\
\hline & spr-04 & $19.27 \pm 0.05$ & $4.89 \pm 0.04$ & $6.78 \pm 0.02$ & $125.67 \pm 0.57$ & $0.52 \pm 0.5$ \\
\hline & sum-04 & $20.27 \pm 0.15$ & $1.86 \pm 0.07$ & $6.44 \pm 0.00$ & $134.67 \pm 0.57$ & $0.60 \pm 0.00$ \\
\hline & aut-04 & $20.53 \pm 0.15$ & $3.29 \pm 0.00$ & $6.64 \pm 0.01$ & $169.33 \pm 1.52$ & $0.47 \pm 0.06$ \\
\hline & win-05 & $17.80 \pm 0.20$ & $2.89 \pm 0.01$ & $6.70 \pm 0.02$ & $166.67 \pm 0.58$ & $0.40 \pm 0.00$ \\
\hline \multirow[t]{4}{*}{ TS4 } & win-03 & 18.80 & 5.75 & 6.19 & 228.00 & \\
\hline & sum-03 & $20.63 \pm 0.75$ & $4.50 \pm 0.17$ & $5.67 \pm 0.06$ & $225.00 \pm 3.35$ & $0.55 \pm 0.01$ \\
\hline & aut-03 & $19.50 \pm 0.09$ & $2.70 \pm 0.05$ & $6.02 \pm 0.07$ & $214.67 \pm 8.38$ & $0.54 \pm 0.05$ \\
\hline & win-04 & $18.27 \pm 0.05$ & $1.57 \pm 0.05$ & $7.64 \pm 0.06$ & $233.33 \pm 3.05$ & $0.61 \pm 0.06$ \\
\hline \multirow[t]{7}{*}{4351} & win-03 & 17.30 & 3.54 & 6.70 & 950.00 & \\
\hline & spr-03 & $20.55 \pm 0.35$ & $3.08 \pm 0.05$ & $6.29 \pm 0.01$ & $374.66 \pm 1.06$ & $0.53 \pm 0.00$ \\
\hline & sum-03 & $19.57 \pm 0.05$ & $0.67 \pm 0.48$ & $6.16 \pm 0.05$ & $232.67 \pm 1.15$ & $1.42 \pm 0.13$ \\
\hline & aut-03 & $19.23 \pm 0.15$ & $3.00 \pm 0.34$ & $7.09 \pm 0.21$ & $147.67 \pm 10.96$ & $0.53 \pm 0.04$ \\
\hline & win-03 & 18.20 & 2.68 & 7.82 & 1668.00 & \\
\hline & win-04 & $18.33 \pm 0.15$ & $1.63 \pm 0.05$ & $7.55 \pm 0.06$ & $7020.00 \pm 30.69$ & $19.97 \pm 0.06$ \\
\hline & win-05 & $19.60 \pm 0.00$ & $2.19 \pm 0.01$ & $7.84 \pm 0.01$ & $16400.00 \pm 10.00$ & $10.00 \pm 0.00$ \\
\hline \multirow[t]{4}{*}{ TS2 } & win-03 & 18.80 & 4.73 & 5.88 & 278.00 & \\
\hline & sum-03 & $20.77 \pm 0.25$ & $2.77 \pm 0.15$ & $5.78 \pm 0.01$ & $275.00 \pm 0.61$ & $0.64 \pm 0.04$ \\
\hline & aut-03 & $19.27 \pm 0.05$ & $1.46 \pm 0.13$ & $6.35 \pm 0.14$ & $279.00 \pm 1.25$ & $0.44 \pm 0.12$ \\
\hline & win-04 & $17.03 \pm 0.32$ & $3.93 \pm 0.15$ & $5.94 \pm 0.06$ & $170.33 \pm 0.70$ & $1.91 \pm 0.08$ \\
\hline \multirow[t]{6}{*}{ sO2a } & spr-03 & $18.70 \pm 0.14$ & $2.05 \pm 0.07$ & $6.62 \pm 0.03$ & $148.67 \pm 0.05$ & $0.53 \pm 0.00$ \\
\hline & aut-03 & $21.40 \pm 0.14$ & $1.71 \pm 0.00$ & $7.02 \pm 0.01$ & $188.33 \pm 1.52$ & $1.05 \pm 0.05$ \\
\hline & spr-04 & $17.77 \pm 0.15$ & $2.51 \pm 0.02$ & $6.92 \pm 0.01$ & $161.33 \pm 1.98$ & $0.99 \pm 0.08$ \\
\hline & sum-04 & $19.70 \pm 0.09$ & $2.11 \pm 0.01$ & $6.71 \pm 0.01$ & $244.67 \pm 8.02$ & $0.67 \pm 0.12$ \\
\hline & aut-04 & $19.17 \pm 0.20$ & $1.93 \pm 0.05$ & $6.88 \pm 0.00$ & $202.00 \pm 2.56$ & $0.53 \pm 0.12$ \\
\hline & win-05 & $18.60 \pm 0.10$ & $2.48 \pm 0.05$ & $6.68 \pm 0.01$ & $198.67 \pm 0.58$ & $0.53 \pm 0.11$ \\
\hline
\end{tabular}


Table 2 Continued

\begin{tabular}{|c|c|c|c|c|c|c|}
\hline Piezometer & Season & $\mathrm{T}\left({ }^{\circ} \mathrm{C}\right)$ & $\begin{array}{l}\mathrm{DO} \\
\left(\mathrm{mg} \mathrm{L}^{-1}\right)\end{array}$ & $\mathrm{pH}$ & 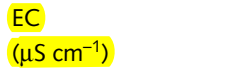 & $\begin{array}{l}\text { Alkalinity } \\
\left(\text { meq } L^{-1}\right)\end{array}$ \\
\hline $4 S 2$ & win-03 & 18.10 & 3.36 & 7.69 & 328.00 & \\
\hline \multirow[t]{6}{*}{$\mathrm{SO} 2 \mathrm{~b}$} & spr-03 & $18.75 \pm 0.35$ & $1.50 \pm 0.28$ & $6.51 \pm 0.07$ & $183.00 \pm 0.26$ & $0.52 \pm 0.00$ \\
\hline & aut-03 & $20.60 \pm 0.01$ & $1.11 \pm 0.00$ & $6.90 \pm 0.03$ & $233.00 \pm 2.56$ & $1.05 \pm 0.04$ \\
\hline & spr-04 & $18.57 \pm 0.05$ & $2.10 \pm 0.25$ & $6.74 \pm 0.02$ & $210.00 \pm 3.58$ & $0.86 \pm 0.26$ \\
\hline & sum-04 & $19.20 \pm 0.33$ & $1.44 \pm 0.00$ & $6.78 \pm 0.02$ & $310.00 \pm 4.21$ & $0.73 \pm 0.12$ \\
\hline & aut-04 & $18.50 \pm 0.17$ & $1.51 \pm 0.01$ & $6.70 \pm 0.00$ & $261.00 \pm 1.25$ & $0.47 \pm 0.12$ \\
\hline & win-05 & $18.60 \pm 0.10$ & $1.60 \pm 0.02$ & $6.56 \pm 0.01$ & $243.00 \pm 1.00$ & $0.60 \pm 0.00$ \\
\hline \multirow[t]{2}{*}{ S50 } & sum-03 & $19.67 \pm 0.05$ & $1.80 \pm 0.10$ & $6.73 \pm 0.06$ & $178.27 \pm 0.05$ & $1.41 \pm 0.14$ \\
\hline & sum-04 & $19.57 \pm 0.15$ & $1.38 \pm 0.05$ & $7.14 \pm 0.02$ & $152.33 \pm 0.57$ & $1.25 \pm 0.04$ \\
\hline S56 & sum-04 & $22.07 \pm 0.11$ & $0.86 \pm 0.00$ & $7.45 \pm 0.03$ & $21010.00 \pm 149.33$ & $6.00 \pm 0.00$ \\
\hline \multirow[t]{7}{*}{ SO1 } & spr-03 & $18.05 \pm 0.19$ & $2.90 \pm 0.00$ & $6.94 \pm 0.07$ & $91.50 \pm 0.01$ & $0.49 \pm 0.00$ \\
\hline & sum-03 & $20.33 \pm 0.15$ & $2.33 \pm 0.20$ & $7.28 \pm 0.09$ & $153.00 \pm 0.75$ & $1.00 \pm 0.09$ \\
\hline & aut-03 & $18.63 \pm 0.05$ & $2.45 \pm 0.06$ & $7.36 \pm 0.12$ & $126.00 \pm 0.02$ & $0.54 \pm 0.04$ \\
\hline & spr-04 & $18.93 \pm 0.05$ & $2.10 \pm 0.08$ & $7.12 \pm 0.02$ & $99.00 \pm 2.36$ & $0.63 \pm 0.04$ \\
\hline & sum-04 & $14.60 \pm 0.10$ & $2.13 \pm 0.01$ & $6.87 \pm 0.00$ & $163.00 \pm 3.69$ & $0.63 \pm 0.06$ \\
\hline & sum-04 & $18.87 \pm 0.15$ & $1.79 \pm 0.01$ & $7.11 \pm 0.01$ & $135.00 \pm 2.56$ & $0.60 \pm 0.00$ \\
\hline & win-05 & $18.50 \pm 0.20$ & $2.20 \pm 0.02$ & $6.67 \pm 0.01$ & $136.00 \pm 0.00$ & $0.47 \pm 0.11$ \\
\hline
\end{tabular}

Table 3 Chemical variables seasonally measured in the piezometers (mean \pm SD) (SRP, soluble reactive phosphorus; win, winter; spr, spring; sum, summer; aut, autumn)

\begin{tabular}{|c|c|c|c|c|c|c|c|c|}
\hline Piezometer & Season & $\begin{array}{l}\text { Ammonium } \\
\left(\mathrm{mg} \mathrm{L}^{-1} \mathrm{~N}-\mathrm{NH}_{4}^{+}\right)\end{array}$ & $\begin{array}{l}\text { Nitrate } \\
\left(\mathrm{mg} \mathrm{L}^{-1} \mathrm{~N}-\mathrm{NO}_{3}\right)\end{array}$ & $\begin{array}{l}\text { SRP } \\
\left(\mathrm{mg} \mathrm{L}^{-1} \mathrm{P}_{-} \mathrm{PO}_{4}^{3-}\right)\end{array}$ & $\begin{array}{l}\text { Total P } \\
\left(m g L^{-1} P\right)\end{array}$ & $\begin{array}{l}\text { Ferric iron } \\
\left(\mathrm{mg} \mathrm{L}^{-1} \mathrm{Fe}^{2+}\right)\end{array}$ & $\begin{array}{l}\text { Ferrous iron } \\
\left(\mathrm{mg} \mathrm{L}^{-1} \mathrm{Fe}^{3+}\right)\end{array}$ & $\begin{array}{l}\mathrm{Fe} \\
\left(\mathrm{mg} \mathrm{L}^{-1} \mathrm{Fe}\right)\end{array}$ \\
\hline \multirow[t]{5}{*}{ Piso } & sum-03 & $0.014 \pm 0.008$ & $1.009 \pm 0.050$ & $0.002 \pm 0.001$ & $0.057 \pm 0.009$ & $0.328 \pm 0.198$ & $0.917 \pm 0.460$ & $1.245 \pm 0.654$ \\
\hline & aut-03 & $0.048 \pm 0.001$ & $0.728 \pm 0.032$ & $0.016 \pm 0.007$ & $0.036 \pm 0.007$ & $0.082 \pm 0.082$ & $0.267 \pm 0.065$ & $0.349 \pm 0.029$ \\
\hline & sum-04 & $0.032 \pm 0.013$ & $1.313 \pm 0.159$ & $0.018 \pm 0.004$ & $0.019 \pm 0.009$ & $0.000 \pm 0.000$ & $0.349 \pm 0.078$ & $0.349 \pm 0.078$ \\
\hline & aut-04 & $0.493 \pm 0.161$ & $0.783 \pm 0.072$ & $0.013 \pm 0.002$ & $0.031 \pm 0.002$ & $0.000 \pm 0.000$ & $0.133 \pm 0.027$ & $0.133 \pm 0.017$ \\
\hline & win-05 & $0.024 \pm 0.012$ & $1.060 \pm 0.108$ & $0.011 \pm 0.003$ & $0.020 \pm 0.006$ & $0.073 \pm 0.122$ & $1.095 \pm 0.914$ & $1.167 \pm 0.867$ \\
\hline \multirow[t]{6}{*}{ S51 } & win-03 & $0.152 \pm 0.012$ & $0.008 \pm 0.001$ & $0.047 \pm 0.008$ & $0.165 \pm 0.003$ & & & \\
\hline & spr-03 & $0.009 \pm 0.000$ & $0.059 \pm 0.060$ & $0.059 \pm 0.004$ & $0.286 \pm 0.042$ & $3.183 \pm 0.746$ & $2.510 \pm 2.063$ & $5.693 \pm 5.810$ \\
\hline & aut-03 & $0.065 \pm 0.001$ & $0.149 \pm 0.007$ & $0.046 \pm 0.008$ & $0.189 \pm 0.096$ & $0.272 \pm 0.471$ & $4.510 \pm 1.666$ & $4.327 \pm 1.199$ \\
\hline & spr-04 & $0.064 \pm 0.022$ & $0.118 \pm 0.012$ & $0.093 \pm 0.008$ & $0.353 \pm 0.042$ & $0.000 \pm 0.000$ & $1.954 \pm 0.649$ & $1.495 \pm 0.649$ \\
\hline & aut-04 & $0.045 \pm 0.031$ & $0.000 \pm 0.000$ & $0.120 \pm 0.025$ & $0.130 \pm 0.017$ & $0.000 \pm 0.000$ & $5.614 \pm 1.329$ & $5.614 \pm 1.329$ \\
\hline & win-05 & $0.006 \pm 0.003$ & $0.192 \pm 0.007$ & $0.239 \pm 0.144$ & $0.247 \pm 0.025$ & $0.080 \pm 0.078$ & $2.083 \pm 1.948$ & $2.163 \pm 1.811$ \\
\hline \multirow[t]{4}{*}{$21 S 3$} & win-03 & $0.160 \pm 0.103$ & $0.008 \pm 0.001$ & $0.008 \pm 0.000$ & $0.042 \pm 0.015$ & & & \\
\hline & spr-03 & $0.010 \pm 0.013$ & $0.001 \pm 0.004$ & $0.000 \pm 0.000$ & $0.030 \pm 0.001$ & $0.700 \pm 0.008$ & $0.592 \pm 0.129$ & $1.292 \pm 0.137$ \\
\hline & sum-03 & $0.005 \pm 0.004$ & $0.054 \pm 0.003$ & $0.012 \pm 0.006$ & $0.040 \pm 0.001$ & $0.645 \pm 0.037$ & $0.509 \pm 0.187$ & $1.153 \pm 0.533$ \\
\hline & aut-03 & $0.044 \pm 0.019$ & $0.107 \pm 0.003$ & $0.011 \pm 0.001$ & $0.042 \pm 0.006$ & $0.048 \pm 0.031$ & $1.663 \pm 0.205$ & $1.712 \pm 0.224$ \\
\hline \multirow[t]{7}{*}{$4 \mathrm{~S} 1$} & spr-03 & $0.016 \pm 0.011$ & $0.024 \pm 0.033$ & $0.001 \pm 0.002$ & $0.024 \pm 0.005$ & $2.030 \pm 0.004$ & $18.604 \pm 2.200$ & $20.635 \pm 2.160$ \\
\hline & sum-03 & $0.019 \pm 0.012$ & $0.031 \pm 0.003$ & $0.000 \pm 0.000$ & $0.043 \pm 0.004$ & $0.122 \pm 0.040$ & $0.654 \pm 0.383$ & $0.776 \pm 0.343$ \\
\hline & aut-03 & $0.047 \pm 0.005$ & $0.108 \pm 0.004$ & $0.003 \pm 0.002$ & $0.025 \pm 0.008$ & $0.000 \pm 0.000$ & $0.888 \pm 0.158$ & $0.888 \pm 0.158$ \\
\hline & aut-04 & $0.058 \pm 0.002$ & $0.248 \pm 0.001$ & $0.013 \pm 0.002$ & $0.062 \pm 0.021$ & $0.000 \pm 0.000$ & $1.647 \pm 0.308$ & $1.647 \pm 0.308$ \\
\hline & sum-04 & $0.038 \pm 0.013$ & $0.495 \pm 0.025$ & $0.006 \pm 0.002$ & $0.019 \pm 0.011$ & $0.119 \pm 0.022$ & $0.853 \pm 0.046$ & $0.972 \pm 0.067$ \\
\hline & aut-04 & $0.053 \pm 0.037$ & $0.004 \pm 0.006$ & $0.006 \pm 0.001$ & $0.028 \pm 0.001$ & $0.000 \pm 0.000$ & $1.655 \pm 0.723$ & $1.655 \pm 0.723$ \\
\hline & win-05 & $0.019 \pm 0.018$ & $0.346 \pm 0.011$ & $0.006 \pm 0.001$ & $0.007 \pm 0.004$ & $0.079 \pm 0.068$ & $2.928 \pm 3.624$ & $3.007 \pm 0.004$ \\
\hline \multirow[t]{2}{*}{ L3 } & sum-03 & $0.029 \pm 0.041$ & $0.170 \pm 0.158$ & $0.004 \pm 0.001$ & $0.027 \pm 0.000$ & $1.024 \pm 0.169$ & $1.692 \pm 1.575$ & $2.715 \pm 1.744$ \\
\hline & spr-04 & $0.049 \pm 0.014$ & $0.112 \pm 0.008$ & $0.009 \pm 0.005$ & $0.083 \pm 0.044$ & $0.000 \pm 0.000$ & $1.702 \pm 0.984$ & $1.702 \pm 0.984$ \\
\hline \multirow[t]{4}{*}{ L7 } & win-03 & $0.005 \pm 0.007$ & $0.069 \pm 0.007$ & $0.008 \pm 0.001$ & $0.031 \pm 0.005$ & & & \\
\hline & sum-03 & $0.000 \pm 0.000$ & $0.203 \pm 0.016$ & $0.007 \pm 0.004$ & $0.040 \pm 0.017$ & $0.658 \pm 0.622$ & $0.693 \pm 0.600$ & $1.351 \pm 1.222$ \\
\hline & sum-04 & $0.458 \pm 0.016$ & $0.204 \pm 0.023$ & $0.016 \pm 0.007$ & $0.087 \pm 0.020$ & $1.047 \pm 0.753$ & $12.910 \pm 3.470$ & $13.957 \pm 2.784$ \\
\hline & aut-04 & $0.309 \pm 0.120$ & $0.384 \pm 0.114$ & $0.026 \pm 0.021$ & $0.085 \pm 0.007$ & $0.000 \pm 0.000$ & $6.355 \pm 1.105$ & $6.255 \pm 1.105$ \\
\hline \multirow[t]{3}{*}{ 39S2 } & win-04 & $0.025 \pm 0.009$ & $1.678 \pm 0.023$ & $0.101 \pm 0.004$ & $0.144 \pm 0.011$ & $0.211 \pm 0.366$ & $0.806 \pm 0.217$ & $1.018 \pm 0.254$ \\
\hline & spr-04 & $0.064 \pm 0.002$ & $1.233 \pm 0.132$ & $0.041 \pm 0.005$ & $0.073 \pm 0.003$ & $0.002 \pm 0.000$ & $2.546 \pm 1.647$ & $2.567 \pm 1.463$ \\
\hline & sum-04 & $0.265 \pm 0.035$ & $2.077 \pm 0.238$ & $0.144 \pm 0.054$ & $0.156 \pm 0.001$ & $0.006 \pm 0.002$ & $0.186 \pm 0.035$ & $0.192 \pm 0.032$ \\
\hline
\end{tabular}

(C) 2008 The Authors

Journal compilation (C) 2008 Blackwell Publishing Ltd 
Table 3 Continued

\begin{tabular}{|c|c|c|c|c|c|c|c|c|}
\hline Piezometer & Season & $\begin{array}{l}\text { Ammonium } \\
\left(\mathrm{mg} \mathrm{L}^{-1} \mathrm{~N}-\mathrm{NH}_{4}^{+}\right)\end{array}$ & $\begin{array}{l}\text { Nitrate } \\
\left(\mathrm{mg} \mathrm{L}^{-1} \mathrm{~N}-\mathrm{NO}_{3}^{-}\right)\end{array}$ & $\begin{array}{l}\text { SRP } \\
\left(\mathrm{mg} \mathrm{L}^{-1} \mathrm{P}_{-} \mathrm{PO}_{4}^{3-}\right)\end{array}$ & $\begin{array}{l}\text { Total P } \\
\left(m g L^{-1} P\right)\end{array}$ & $\begin{array}{l}\text { Ferric iron } \\
\left(\mathrm{mg} \mathrm{L}^{-1} \mathrm{Fe}^{2+}\right)\end{array}$ & $\begin{array}{l}\text { Ferrous iron } \\
\left(\mathrm{mg} \mathrm{L}^{-1} \mathrm{Fe}^{3+}\right)\end{array}$ & $\begin{array}{l}\mathrm{Fe} \\
\left(\mathrm{mg} \mathrm{L} \mathrm{L}^{-1} \mathrm{Fe}\right)\end{array}$ \\
\hline & aut-04 & $0.043 \pm 0.015$ & $1.028 \pm 0.152$ & $0.154 \pm 0.071$ & $0.155 \pm 0.010$ & $0.002 \pm 0.003$ & $0.884 \pm 0.215$ & $0.886 \pm 0.212$ \\
\hline & win-05 & $0.025 \pm 0.005$ & $1.112 \pm 0.444$ & $0.061 \pm 0.008$ & $0.075 \pm 0.007$ & $0.000 \pm 0.000$ & $2.114 \pm 1.719$ & $2.114 \pm 1.719$ \\
\hline \multirow[t]{4}{*}{ TS4 } & win-03 & $0.156 \pm 0.006$ & $11.657 \pm 0.931$ & $0.003 \pm 0.000$ & $0.022 \pm 0.009$ & & & \\
\hline & sum-03 & $0.002 \pm 0.000$ & $9.245 \pm 0.676$ & $0.012 \pm 0.006$ & $0.013 \pm 0.003$ & $0.066 \pm 0.032$ & $0.364 \pm 0.285$ & $0.430 \pm 0.317$ \\
\hline & aut-03 & $0.000 \pm 0.000$ & $12.568 \pm 1.989$ & $0.016 \pm 0.006$ & $0.022 \pm 0.003$ & $0.000 \pm 0.000$ & $0.602 \pm 0.670$ & $0.602 \pm 0.671$ \\
\hline & win-04 & $0.001 \pm 0.003$ & $11.645 \pm 0.493$ & $0.014 \pm 0.006$ & $0.059 \pm 0.009$ & $0.130 \pm 0.125$ & $0.376 \pm 0.076$ & $0.507 \pm 0.245$ \\
\hline \multirow[t]{4}{*}{$43 S 1$} & win-03 & $0.078 \pm 0.054$ & $0.374 \pm 0.054$ & $0.016 \pm 0.000$ & $2.064 \pm 0.063$ & & & \\
\hline & spr-03 & $0.019 \pm 0.000$ & $0.866 \pm 0.781$ & $0.001 \pm 0.001$ & $0.040 \pm 0.006$ & $0.164 \pm 0.039$ & $1.185 \pm 0.725$ & $1.350 \pm 0.764$ \\
\hline & sum-03 & $0.003 \pm 0.000$ & $0.135 \pm 0.008$ & $0.006 \pm 0.001$ & $0.061 \pm 0.031$ & $0.357 \pm 0.057$ & $1.414 \pm 0.899$ & $1.771 \pm 0.906$ \\
\hline & aut-03 & $0.025 \pm 0.017$ & $0.464 \pm 0.053$ & $0.011 \pm 0.003$ & $0.062 \pm 0.008$ & $0.000 \pm 0.000$ & $2.392 \pm 1.102$ & $2.392 \pm 1.102$ \\
\hline \multirow[t]{3}{*}{ S60 } & win-03 & $4.613 \pm 0.019$ & $0.060 \pm 0.000$ & $1.813 \pm 0.014$ & $1.814 \pm 0.023$ & & & \\
\hline & win-04 & $2.215 \pm 0.411$ & $0.348 \pm 0.061$ & $0.012 \pm 0.007$ & $0.073 \pm 0.015$ & $1.016 \pm 1.001$ & $0.747 \pm 0.978$ & $1.762 \pm 2.086$ \\
\hline & win-05 & $7.308 \pm 1.175$ & $0.160 \pm 0.022$ & $1.614 \pm 0.062$ & $1.640 \pm 0.107$ & $0.254 \pm 0.298$ & $1.066 \pm 0.226$ & $1.319 \pm 0.511$ \\
\hline \multirow[t]{4}{*}{ TS2 } & win-03 & $0.136 \pm 0.017$ & $9.591 \pm 2.141$ & $0.028 \pm 0.017$ & $0.067 \pm 0.041$ & & & \\
\hline & sum-03 & $0.034 \pm 0.037$ & $12.226 \pm 1.308$ & $0.020 \pm 0.004$ & $0.065 \pm 0.024$ & $0.266 \pm 0.175$ & $0.741 \pm 0.242$ & $1.007 \pm 0.139$ \\
\hline & aut-03 & $0.031 \pm 0.014$ & $19.220 \pm 0.284$ & $0.008 \pm 0.006$ & $0.051 \pm 0.016$ & $0.000 \pm 0.000$ & $1.010 \pm 0.297$ & $1.010 \pm 0.297$ \\
\hline & win-04 & $0.000 \pm 0.000$ & $4.565 \pm 0.294$ & $0.041 \pm 0.007$ & $0.093 \pm 0.011$ & $0.136 \pm 0.035$ & $0.392 \pm 0.068$ & $0.528 \pm 0.251$ \\
\hline \multirow[t]{6}{*}{$\mathrm{SO} 2 \mathrm{a}$} & spr-03 & $0.007 \pm 0.001$ & $0.780 \pm 0.703$ & $0.023 \pm 0.003$ & $0.024 \pm 0.003$ & $0.040 \pm 0.021$ & $0.361 \pm 0.170$ & $0.401 \pm 0.191$ \\
\hline & aut-03 & $0.010 \pm 0.010$ & $0.515 \pm 0.065$ & $0.038 \pm 0.005$ & $0.079 \pm 0.012$ & $0.003 \pm 0.004$ & $0.424 \pm 0.082$ & $0.427 \pm 0.081$ \\
\hline & spr-04 & $0.039 \pm 0.013$ & $0.717 \pm 0.009$ & $0.031 \pm 0.002$ & $0.058 \pm 0.012$ & $0.000 \pm 0.000$ & $1.018 \pm 0.696$ & $1.018 \pm 0.696$ \\
\hline & sum-04 & $0.000 \pm 0.000$ & $1.114 \pm 0.095$ & $0.033 \pm 0.001$ & $0.040 \pm 0.008$ & $0.005 \pm 0.005$ & $1.343 \pm 0.696$ & $1.348 \pm 0.218$ \\
\hline & aut-04 & $0.000 \pm 0.000$ & $0.411 \pm 0.043$ & $0.037 \pm 0.002$ & $0.053 \pm 0.002$ & $0.000 \pm 0.000$ & $0.263 \pm 0.134$ & $0.263 \pm 0.134$ \\
\hline & win-05 & $0.000 \pm 0.000$ & $0.535 \pm 0.090$ & $0.035 \pm 0.001$ & $0.037 \pm 0.000$ & $0.030 \pm 0.029$ & $0.620 \pm 0.610$ & $0.650 \pm 0.591$ \\
\hline $4 \mathrm{~S} 2$ & win-03 & $0.273 \pm 0.014$ & $0.009 \pm 0.000$ & $0.009 \pm 0.009$ & $0.085 \pm 0.009$ & & & \\
\hline \multirow[t]{6}{*}{$\mathrm{SO} 2 \mathrm{~b}$} & spr-03 & $0.000 \pm 0.000$ & $0.671 \pm 0.605$ & $0.008 \pm 0.001$ & $0.015 \pm 0.000$ & $0.030 \pm 0.005$ & $0.328 \pm 0.181$ & $0.331 \pm 0.176$ \\
\hline & aut-03 & $0.017 \pm 0.003$ & $0.199 \pm 0.005$ & $0.016 \pm 0.000$ & $0.048 \pm 0.009$ & $0.000 \pm 0.000$ & $0.613 \pm 0.571$ & $0.613 \pm 0.791$ \\
\hline & spr-04 & $0.023 \pm 0.011$ & $0.334 \pm 0.038$ & $0.017 \pm 0.003$ & $0.022 \pm 0.004$ & $0.000 \pm 0.000$ & $0.503 \pm 0.361$ & $0.503 \pm 0.361$ \\
\hline & sum-04 & $0.027 \pm 0.004$ & $0.999 \pm 0.011$ & $0.015 \pm 0.002$ & $0.020 \pm 0.005$ & $0.004 \pm 0.005$ & $0.239 \pm 0.080$ & $0.242 \pm 0.075$ \\
\hline & aut-04 & $0.000 \pm 0.000$ & $0.222 \pm 0.039$ & $0.013 \pm 0.002$ & $0.044 \pm 0.007$ & $0.000 \pm 0.000$ & $0.077 \pm 0.036$ & $0.077 \pm 0.036$ \\
\hline & win-05 & $0.000 \pm 0.000$ & $0.250 \pm 0.162$ & $0.014 \pm 0.000$ & $0.015 \pm 0.001$ & $0.040 \pm 0.054$ & $0.546 \pm 0.454$ & $0.586 \pm 0.437$ \\
\hline \multirow[t]{2}{*}{ S50 } & sum-03 & $0.000 \pm 0.000$ & $0.134 \pm 0.001$ & $0.022 \pm 0.002$ & $0.038 \pm 0.011$ & $0.029 \pm 0.007$ & $0.166 \pm 0.030$ & $0.195 \pm 0.029$ \\
\hline & sum-04 & $0.015 \pm 0.001$ & $0.261 \pm 0.017$ & $0.029 \pm 0.000$ & $0.033 \pm 0.006$ & $0.001 \pm 0.000$ & $0.408 \pm 0.168$ & $0.408 \pm 0.168$ \\
\hline S56 & sum-04 & $7.728 \pm 1.385$ & $0.431 \pm 0.035$ & $0.423 \pm 0.041$ & $0.521 \pm 0.041$ & $0.123 \pm 0.083$ & $1.317 \pm 0.725$ & $1.440 \pm 0.649$ \\
\hline \multirow[t]{7}{*}{ SO1 } & spr-03 & $0.000 \pm 0.000$ & $0.622 \pm 0.562$ & $0.007 \pm 0.000$ & $0.026 \pm 0.004$ & $0.000 \pm 0.000$ & $0.232 \pm 0.067$ & $0.232 \pm 0.067$ \\
\hline & sum-03 & $0.034 \pm 0.029$ & $0.223 \pm 0.019$ & $0.028 \pm 0.003$ & $0.032 \pm 0.005$ & $0.425 \pm 0.197$ & $1.177 \pm 0.655$ & $1.602 \pm 0.725$ \\
\hline & aut-03 & $0.031 \pm 0.007$ & $0.571 \pm 0.020$ & $0.023 \pm 0.002$ & $0.040 \pm 0.014$ & $0.000 \pm 0.000$ & $5.832 \pm 4.963$ & $5.832 \pm 8.963$ \\
\hline & spr-04 & $0.038 \pm 0.012$ & $0.466 \pm 0.049$ & $0.019 \pm 0.002$ & $0.031 \pm 0.011$ & $0.000 \pm 0.000$ & $0.318 \pm 0.178$ & $0.318 \pm 0.178$ \\
\hline & sum-04 & $0.000 \pm 0.000$ & $0.879 \pm 0.083$ & $0.025 \pm 0.001$ & $0.035 \pm 0.007$ & $0.011 \pm 0.005$ & $0.158 \pm 0.018$ & $0.169 \pm 0.023$ \\
\hline & sum-04 & $0.023 \pm 0.018$ & $0.540 \pm 0.024$ & $0.027 \pm 0.009$ & $0.045 \pm 0.008$ & $0.000 \pm 0.000$ & $0.227 \pm 0.026$ & $0.227 \pm 0.026$ \\
\hline & win-05 & $0.000 \pm 0.000$ & $0.151 \pm 0.009$ & $0.024 \pm 0.002$ & $0.034 \pm 0.003$ & $0.018 \pm 0.032$ & $0.478 \pm 0.441$ & $0.496 \pm 0.426$ \\
\hline
\end{tabular}

A significant increase in bacterial biomass was seasonally observed during 2003 in all wells, with higher values during summer or autumn than during winter or spring $(P \leq 0.008)$, 7 except in borehole SO2a $(P \geq 0.378)$. During 2004 , a similar 8 temporal pattern was observed $(P \leq 0.023)$, except in well 9 SO2a $(P \geq 0.567)$. Bacterial biomass positively correlated 50 with temperature $(r=0.471, P=0.000, n=71)$ and nitrate $51(r=0.423, P=0.000, n=71)$, and negatively with DO $52(r=-0.276, P=0.021, n=71)$. In wells S51, SO2b and 53 SOl bacterial biomass negatively correlated with rainfall 54 from 2 months prior to sampling $(P \leq 0.035)$. This variable did not correlate with depth, permeability or transmissivity in any season.

Microbial activities of functional groups, except those for $\mathrm{NB}$, were found in this aquifer system. Significant differences for the activities of different functional groups, measured as the mean of the first day after incubation in which activity was detected, were observed in all seasons $(F \geq 5.530, P \leq 0.009)$ (Table 4). During 2003 and winter 2004, IRB showed statistically higher activities than SRB and DN $(P \leq 0.001)$, whose activities were not significantly different $(P \geq 0.099)$. In summer 2004, IRB and SRB showed statistically similar 

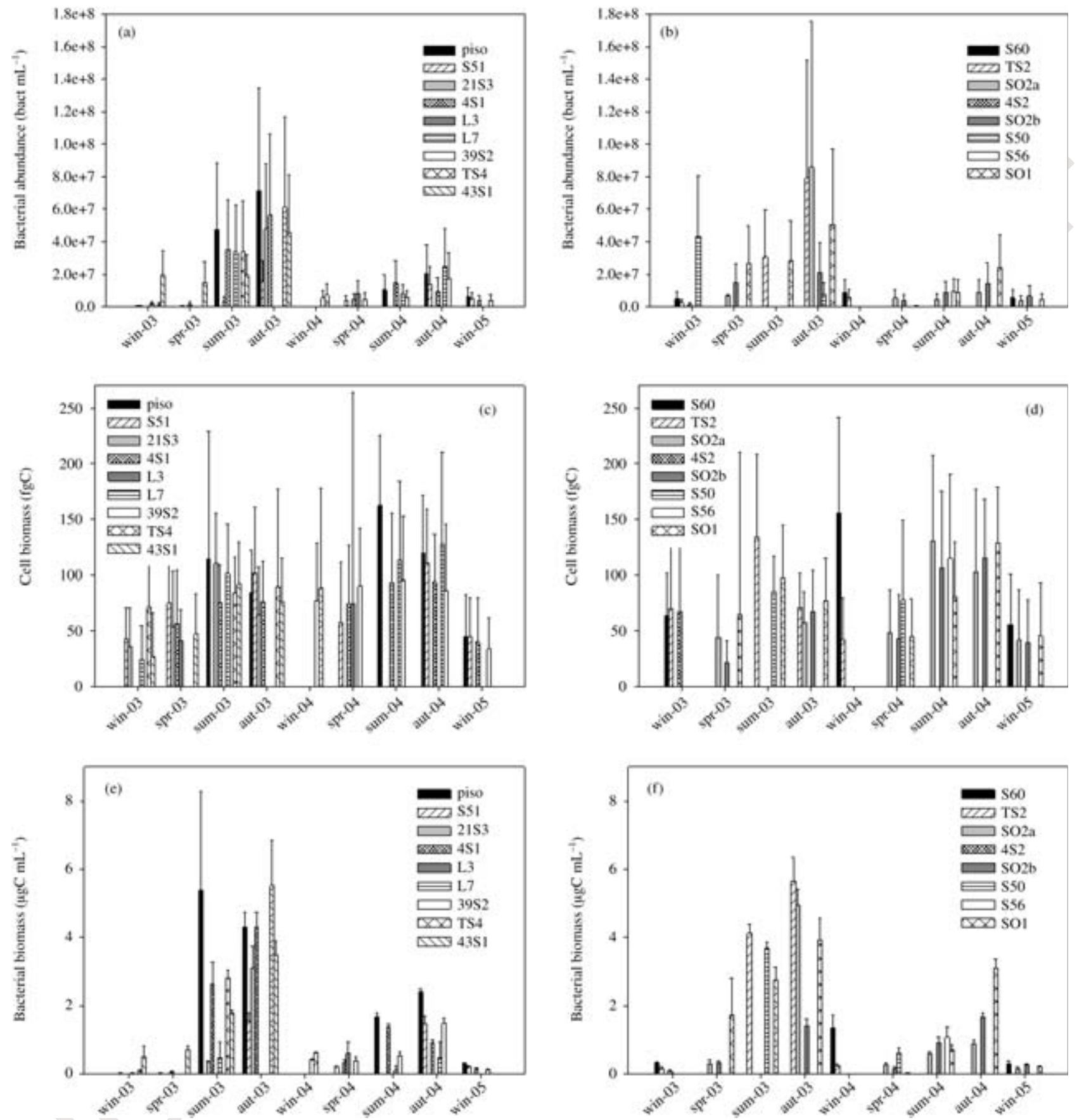

Fig. 2 Seasonal changes in microbiological variables during the study period: a and b, bacterial abundance, $c$ and d, cell biomass, e and $f$, bacterial biomass (win, winter; spr, spring; sum, summer; aut, autumn; solid bars denote standard deviation).

activities $(P \geq 0.123)$ and significantly higher than $\mathrm{DN}(P \leq 0.009)$. During 2003, higher activities in summer than in winter were found in all cases, although they were only significant for IRB and SRB activities in some wells (21S3, L7 and TS2; $P \leq 0.035$ ). In general, there were not significant differences in the means of microbial activities between summer and winter during 2004. No significant correlations were found among microbial activities, depth, permeability and transmissivity.

\section{Exploratory statistical analyses}

Bartlett's $\chi^{2}$ test showed that the two sets of variables (microbiological and physicochemical) in the canonical correlation analysis were not independent $\left(\chi^{2}=60.985\right.$, d.f. $\left.=39, P=0.014\right)$; the first two canonical variate pairs were significantly correlated (Table 5). The strong correlation observed for the first canonical variate pair symbolizes a correlation between a variate that 
Table 4 Summary of microbial activities for different functional groups provided by BART ${ }^{\mathrm{TM}}$.tests (DN, denitrifying bacteria; IRB, iron-related bacteria; SRB, sulphatereducing bacteria; win, winter; sum, summer). Numbers indicate first day in which bacterial activity was recorded

\begin{tabular}{|c|c|c|c|c|c|c|c|c|c|c|c|c|}
\hline \multirow[b]{2}{*}{ ezometer } & \multicolumn{4}{|l|}{ DNB } & \multicolumn{4}{|l|}{ IRB } & \multicolumn{4}{|l|}{ SRB } \\
\hline & win-03 & sum-03 & win-04 & sum-04 & win-03 & sum-03 & win-04 & sum-04 & win-03 & sum-03 & win-04 & sum-04 \\
\hline so & & 10 & & 3 & & 4 & & 2 & & 4 & & 9 \\
\hline 1 & 6 & & & & 3 & & & & 10 & & & \\
\hline S3 & 5 & 3 & & & 10 & 4 & & & 10 & 10 & & 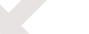 \\
\hline 1 & & 9 & & 2 & & 2 & & 3 & & 5 & & 3 \\
\hline & 10 & 10 & & 2 & 4 & 3 & & 2 & 7 & 5 & & 7 \\
\hline S2 & & & 10 & 10 & & & 3 & 2 & & & 9 & 4 \\
\hline 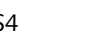 & 8 & 4 & 4 & & 4 & 3 & 4 & & 10 & 6 & 10 & \\
\hline S1 & & 10 & & & & 4 & & & & 6 & & \\
\hline 0 & 5 & & 4 & & 1 & & 1 & & 3 & & 4 & \\
\hline 2 & 4 & 4 & 4 & & 3 & 1 & 4 & & 8 & 5 & 8 & \\
\hline $2 a$ & & & & 3 & & & & 3 & & & & 6 \\
\hline 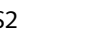 & 7 & & & & 4 & & & & 10 & 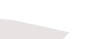 & & \\
\hline $2 b$ & & & & 3 & & & & 2 & & & & 4 \\
\hline 0 & & 6 & & & & 2 & & & & 8 & & \\
\hline 6 & & & & 1 & & & & 2 & & & & 5 \\
\hline 1 & & 10 & & 6 & & 3 & & 10 & & 5 & & 3 \\
\hline
\end{tabular}

Table 5 Results after Bartlett's $\chi^{2}$ test with successive variate pair removed for the canonical correlation analysis

\begin{tabular}{llllll}
\hline Variate pair removed & Canonical $R$ & Canonical $R^{2}$ & $\chi^{2}$ & d.f. & $P$ \\
\hline 0 & 0.710 & 0.504 & 80.709 & 39 & 0.000 \\
1 & 0.557 & 0.310 & 37.534 & 24 & 0.039 \\
2 & 0.461 & 0.212 & 14.673 & 11 & 0.198 \\
\hline
\end{tabular}

combines temperature, DO, nitrate, ferrous iron and total iron, and another variate that integrates bacterial abundance, cell biomass and bacterial biomass (Fig. 3). Bacterial abundances, and mainly, cell and bacterial biomasses increase with higher temperatures and nitrate concentrations but, with higher DO, ferrous ion and total iron concentrations, bacterial abundances, cell biomasses and bacterial biomasses show a decrease. The correlation observed for the second canonical variate pair was less significant (Table 5 ). The variance explained by the first three factors included in the PCA of the correlation matrix performed was $70.21 \%$. Microbiological variables, temperature and nitrate concentration defined the principal component I. Samples were classified into two major groups in relation to axis I: one on the positive side (winter/spring 2003, winter/ spring 2004 and winter 2005) and another on the negative side (summer/autumn 2003, and summer/autumn 2004) (Fig. 4).

\section{DISCUSSION}

\section{Physicochemical variables}

As surface waters, whose temperature and DO values display temporal patterns (Coleto, 2003), groundwater temperature and DO also exhibited temporal patterns in the aquifer system of Doñana. In other aquifer systems, no significant seasonal differences for temperature, at least between winter and summer, were observed (Balkwill et al., 1989; Pedersen et al., 1996). This aquifer system therefore appears to be very different to other subsurface systems considered to be highly stable (Vorobyova et al., 1997). The decrease in groundwater DO from winter to autumn during both years might be due to inorganic reactions involving reduced metals, microbial utilization of reduced organic matter, or both (Phelps et al., 1994; Brugger et al., 2001; López-Archilla et al., 2007). Organic matter concentration in the Doñana aquifer is moderately high (Coleto, 2003); as a result, low DO concentrations found in autumns might be due to microbial consumption (Griebler et al., 2002). Electric conductivity was higher in wells located close to the marsh because there is a clear influence of brackish water. $\mathrm{pH}$ was close to neutral and, due to the absence of correlations between this chemical variable and the microbiological variables, we suggest that $\mathrm{pH}$ was not a key factor controlling the spatiotemporal distribution of microbial communities present in this aquifer system, as has been previously pointed out (Gounot, 1996); however, $\mathrm{pH}$ was a central factor controlling the diversity and the taxonomic composition of microbial communities in Doñana's groundwater (López-Archilla et al., 2007).

Although aquifers have often been considered to be oligotrophic environments (Mikell et al., 1996) more similar to open marine systems than to lakes or shallow lakes, and with bacterial densities close to those observed for oceans (Pedersen \& Ekendahl, 1990), we consider that these ideas can neither be generalized nor extrapolated. Doñana's aquifer system, at least its upper unit, appears to be meso- or eutrophic if concentrations of nitrogen and phosphorus inorganic chemical 
Fig. 3 Canonical correlation analysis showing physicochemical variables versus microbiological variables form the first variate pair (A). Contributions of the descriptors to the first variate pair (B) (boldface type indicates the major canonical loadings in the first variate pair). DO, dissolved oxygen; EC, electric conductivity; SRP, soluble reactive phosphorus; TP, total phosphorus.

Fig. 4 Positions of the 71 seasonal samples plotted in the reduced space of the first two principal components, showing both the 10 descriptors or the variables projected on the plane determined by the first two principal axes (box a) and the factor loadings of these descriptors on each of the first two axes (box b) (boldface type indicates the major factor loadings on each axis). DO, dissolved oxygen; SRP, soluble reactive phosphorus.
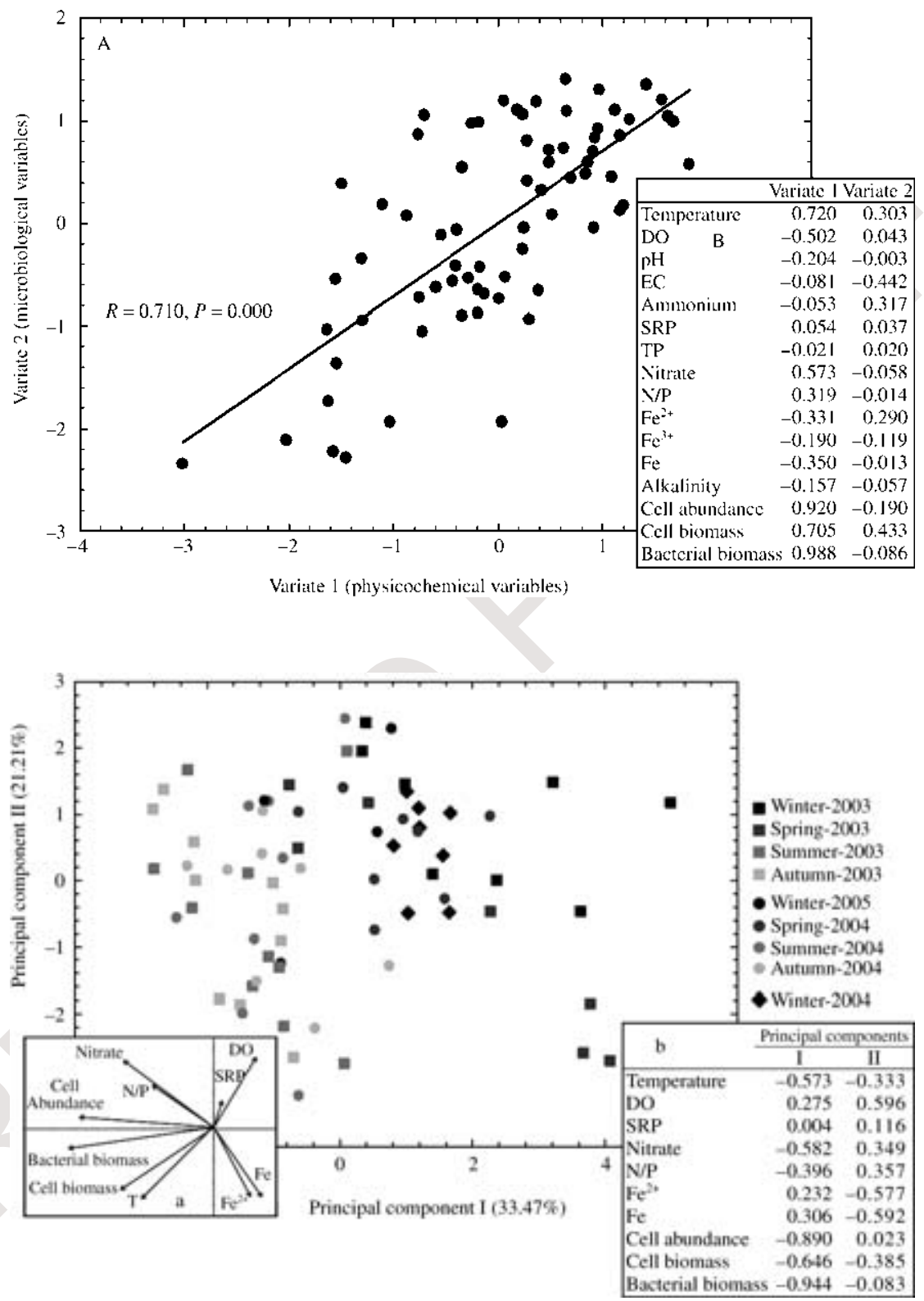

forms are considered (Table 3 ). Although several wells were used in this study, and spatiotemporal variability is notable, ammonium, nitrate, SRP and TP concentrations were higher than, or at least similar to, those values reported for other aquifer systems (Alfreider et al., 1997; Murphy \& Schramke, 1998; Trojan et al., 2003; Mehnert et al., 2007). Most of the wells in this study showed higher concentrations for nitrate than for ammonium, probably because most of them were well oxygenated during the study period (Tables 2 and 3 ). Nitrate concentrations are usually low in groundwaters, unless there is a pollution source from agricultural fertilizers (Gounot, 1996). In this study, high nitrate concentrations found in wells TS2 and TS4 are explained by the presence of intensive strawberry crops (Manzano et al., 2007). A high iron concentration in the groundwater is explained by the presence of an intermediate layer with iron oxide minerals (Trick \& Custodio, 2004). High total iron concentrations, as well as ferrous and ferric iron concentrations, had a considerable influence on the type of microorganisms found in this ecosystem (López-Archilla et al., 2007). At the same time, high concentrations of organic matter, that can act as a source of electrons for microorganisms, were also determined in this aquifer system (Coleto, 2003). Both ferric iron and nitrate can act as electron acceptors when DO is depleted. In fact, IRB have displayed the highest activities, highlighting the relative importance of iron (and its chemical forms) in the general microbial metabolism of this aquifer 
system. Although high nitrate concentrations were found in this aquifer system, however, DNB exhibited lower activity levels than IRB and SRB, demonstrating that there are no reasons to consider linear relationships among certain chemical forms, used as electron acceptors by microorganisms, and functional groups (Mauck \& Roberts, 2007), because aquifer heterogeneity results in a heterogenerous distribution of the microbial communities and their activities (Goldscheider $e t$ al., 2006). In any case, the activity of microbial communities is probably influencing the geochemistry of this aquifer system, as has been observed elsewhere (Bennett et al., 2000; Chapelle, 2000a; Penny et al., 2003; Dassonville et al., 2004; Haack et al., 2004; Roadcap et al., 2006).

\section{Microbiological variables}

Prokaryotes appear to dominate the microbial communities in this aquifer system. Eukaryotes were not detected in microscopic counts. This is not surprising because, although the presence of algae, protozoa and fungi could be important in some aquifer ecosystems, prokaryotes represent, by far, the most abundant and diverse microbial group in aquifers, at least in the phreatic zone (Balkwill, 1989; Sinclair \& Ghiorse, 1989; Whitman et al., 1998).

Short rod-shaped bacteria have some advantages over large rod-shaped and filamentous bacteria for transport through sandy sediments (Harvey et al., 1984). Data in this study confirm previous ones (Velasco et al., 2008; in press) and demonstrate that mean bacterial abundance observed in this aquifer system was, at least, one order of magnitude higher than that found in other sedimentary and relatively similar aquifer ecosystems (Harvey et al., 1984; Kölbel-Boelke et al., 1988; Marxsen, 1988; Hirsch \& Rades-Rohkohl, 1990; Hazen et al., 1991; Alfreider et al., 1997; Griebler et al., 2002). Groundwater mean bacterial abundance found in this study was also higher than planktonic bacterial densities found in granite (Eydal \& Pedersen, 2007) or rock aquifer systems (Lehman et al., 2004). Indeed, planktonic bacterial abundance in the Doñana aquifer was close to values reported for attached bacteria in other sedimentary aquifer systems (Alfreider et al., 1997; Kieft et al., 1998; Martino et al., 1998). Several comparative studies have shown that attached bacteria exhibit more density than unattached bacteria in sedimentary aquifers (Alfreider et al., 1997), as well as in rock aquifers (Lehman et al., 2004). Bearing in mind that our data only show the density of planktonic bacteria, the real bacterial abundance of Doñana's aquifer system might well be higher. However, the abundance of unattached bacteria can be significant in shallow, eutrophic and sedimentary aquifer systems (Harvey et al., 1984; Harvey \& George, 1987; Bengtsson, 1989; Griebler et al., 2002).

Average cell biomass values were similar to those found in other sandy sediments (Bone \& Balkwill, 1988), but were slightly higher than a value reported for a sedimentary, sandy and very similar aquifer system (Marxsen, 1988). Bacterial biomass values were in the same order of magnitude as those found in other sandy, aquifer systems (Marxsen, 1988).

\section{Distribution of microbial communities in the aquifer system}

Differences in bacterial abundance among wells sampled during the same season were not significant in most sampling campaigns, as has been observed elsewhere (Kölbel-Boelke et al., 1988). Moreover, differences for cell and bacterial biomass among wells were not great during some seasons. In sandy aquifer systems, spatial heterogeneity can be more important for sediments than for groundwaters on a small scale (Fredrickson et al., 1991; Brockman \& Murray, 1997), in spite of the homogeneity of lithologies that can occur on a greater scale (Zhou et al., 2004). Consequently, microbiological variables could present fewer and less significant differences among groundwater samples than among sediment core samples (Kölbel-Boelke et al., 1988). Moreover, differences among replicates of the same aquifer sample point can be higher than differences among different aquifer sample points (Brockman \& Murray, 1997). In other studies, no differences in bacterial abundance were found, either in groundwaters (Pedersen \& Ekendahl, 1990) or in sediment core samples (Hazen et al., 1991). Finally, it should be borne in mind that field samples represent the summation of a complex series of environmental interactions acting over vastly different temporal and spatial scales, being difficult to find clear spatiotemporal patterns in microbial communities (Shi et al., 1999).

Although it was presumed that bacterial abundance decreases with depth, in general, no obvious correlations between these variables have been found in shallow, sedimentary aquifer systems (Beloin et al., 1988; Sinclair \& Ghiorse, 1989; Phelps et al., 1994; Fredrickson et al., 1997; Martino et al., 1998). Negative relationships have been observed, however, between these two variables in the vadose zone of some low recharge aquifer systems (Fredrickson et al., 1997; Kieft et al., 1998). In Doñana's aquifer system, no significant correlations were detected between depth and bacterial abundance during any season, with exceptions in some deep wells. Moreover, no significant correlations were found between cell biomass and depth during any season in the Doñana aquifer system. Consequently, depth does not seem to be a key factor controlling microbial communities in terms of abundance and biomass in this aquifer system, at least in the range of depths studied. Furthermore, no significant correlations were found between microbial functional groups and depth, although conclusions should be established with care because the number of samples was low $(n \leq 8)$.

Grain size has often been considered to be one of the most important factors controlling microbial abundance and activity in aquifer systems (Musslewhite et al., 2003). Layers with higher clay content usually exhibit lower attached bacterial abundances than sandy layers (Fredrickson et al., 1997; Musslewhite et al., 2003), although positive correlations between 
1 sediment clay content and bacterial density have also been 2 observed (Balkwill, 1989), showing that we should emphasize 3 the need to consider site-specific environmental factors in 4 order to understand microbial distribution and activity (Kieft 5 et al., 1998). In spite of the fact that subsurface sediments and 6 groundwaters represent different milieus (Madsen \& Ghiorse, 7 1993), grain size might also affect the planktonic microbial 8 communities because there is a permanent cell exchange between 9 suspended and attached bacteria (Hirsch \& Rades-Rohkohl, 10 1990; Madsen \& Ghiorse, 1993). In the aquifer system of 11 Doñana, piezometers with the finest materials (high clay 12 contents) in the screen region (TS2 and 4S2) (Table 1) 13 showed lower bacterial abundances than boreholes with 14 coarser materials (medium sands or coarse sands), although no 15 significant differences were found. Moreover, wells $21 S 3$ and 16 S51, with fine sands in the screen region, showed lower bacterial 17 densities, often with significant differences, than other piezometers 18 with coarser lithologies (Fig. 2 A,B) and borehole L7, with coarse 19 sands in the screen region, displayed high bacterial abundances 20 during three sampling seasons. Consequently, a clearer relation21 ship between grain size and bacterial abundance than between 22 depth and bacterial abundance was observed in the Doñana 23 aquifer system. Nonetheless, differences for cell biomasses 24 between wells with fine materials and wells with coarse materials 25 were less evident. Finally, no significant or clear patterns were 26 found between the microbial activities of functional groups and 27 grain size, although conclusions should be reached with care 28 because only a few samples were considered $(n \leq 5)$.
The apparent control of grain size over microbial communities might be due to some hydrogeological parameters, such as permeability, porosity or transmissivity (Brockman \& Murray, 1997; Musslewhite et al., 2003). Areas that show higher hydraulic conductivities tend to display higher bacterial biomasses (Fredrickson et al., 2004) and activities (Chapelle \& Lovley, 1990), because this variable determines the hydrological flows and, consequently, the nutrient supplies to bacteria (Lehman et al., 2001; Zhou et al., 2004) and the movement of cells through the aquifer system (Balkwill et al., 1998). It seems that clay content, per se, may not directly control microbial population densities in the subsurface, but rather the influence of clay on microbial populations in the subsurface may be due to the effect that clay has on hydraulic conductivity or water activity (Fredrickson et al., 1991). However, no correlations between microbiological and hydrogeological variables (permeability and transmissivity) were observed in this study. Considering the grain size homogeneity reported at least for the upper unit of this aquifer system (Trick \& Custodio, 2004), where most of the sampled piezometers are located, a detailed hydrogeological study would contribute to clarifying the spatial distribution of their microbial communities. A common project between microbiologists and hydrogeologists is desirable, but only if both share the same spatiotemporal scale (Brockman \& Murray, 1997).

Moreover, and taking into account the correlations found between microbiological variables and rainfall, hydrology, determined by rainfall, could exert an important control over the microbial communities of this aquifer system, mainly if rainfall is considered to be the only source of freshwater in the aquifer system and, as a result, largely determines hydrogeological flows and phreatic levels (Trick \& Custodio, 2004). Bacterial abundance and cell biomass negatively correlated with rainfall 2 months prior to sampling in wells SO1, SO2a, SO2b and S51. Curiously, these wells are located close to the lower unit, where important hydrological horizontal flows occur (SO1, SO2a and $\mathrm{SO} 2 \mathrm{~b}$ ), or close to the ecotone, the most important discharge area of the aquifer system (S5l). As a consequence, it seems that hydrology has a relatively significant influence over microbial communities in areas where regional hydrogeological flows are significant. However, other wells located in the ecotone (21S3) or close to other important discharge areas of the aquifer (4S1, 4S2 and L3) did not correlate with rainfall, which suggests that there are other variables, probably also related to hydrogeology, controlling the distribution of microbial communities in this aquifer system.

Significant correlations between microbiological and physicochemical variables in sedimentary aquifers (Martino et al., 1998; Musslewhite et al., 2003; Santoro et al., 2006) are scarce, because aquifer microbial communities are controlled by environmental attributes that are more difficult to quantify, such as microscale spatial heterogeneity and temporal variability of geochemical parameters (Kölbel-Boelke et al., 1988; Fredrickson et al., 1991; Brockman \& Murray, 1997; Santoro et al., 2006; Mauck \& Roberts, 2007). However, some correlations have been observed between groundwater physicochemical variables and microbial communities in crystalline rock aquifers (Pedersen \& Ekendahl, 1990). In the present study, a canonical correlation analysis showed a strong correlation between a variate integrating nitrate, ferrous iron and total iron and a variate combining bacterial abundance and both cell and bacterial biomass (Fig. 3). Nitrate concentrations are usually low in aquifers unless a source from agricultural fertilizers exists (Gounot, 1996; Brugger et al., 2001), and this is the case observed for some piezometers (Table 1) located in the vicinity of strawberry crops in Doñana. Indeed, three of five piezometers affected by high nitrate concentrations (TS2, TS4 and L7) showed high or the highest bacterial abundance and cell biomass values during some seasons. Relationships among microbiological variables, ferrous iron and total iron were not so clear, but the ferric iron might probably be used as an electron acceptor when DO is depleted (McLean et al., 2006).

\section{Temporal pattern}

A temporal pattern was observed in the microbial communities of this aquifer system, mainly during 2003, but also during 2004 , with highest bacterial abundances, cell biomasses and bacterial biomasses during the warmest months of the year (Fig. 4). Similar results were observed in a parallel study (Velasco et al., 2008; in press). Temperature correlated with these three microbiological variables and seemed to be the 
most important physical factor controlling them throughout 2 the year. Light increases of this variable triggered microbiological processes, including functional groups. However, the temporal pattern was not clear in deep wells ( $\mathrm{SO} 1, \mathrm{SO} 2 \mathrm{a}$ and $\mathrm{SO} 2 \mathrm{~b}$ ). As a consequence, in deep areas of Doñana's aquifer system there are probably other factors, perhaps more related to hydrogeology than to temperature, controlling the temporal distribution of the microbial communities. Seasonal variations of microbial communities in other aquifer systems have also been described (Balkwill \& Ghiorse, 1985; Beloin et al., 1988; Bone \& Balkwill, 1988). Bacterial abundance also correlated with cell biomass in this study, demonstrating a close relationship between the increase in bacterial abundance and the amount of accumulated carbon in cells (Kieft et al., 1998).

\section{CONCLUSIONS}

Although it would be incorrect to depict general conclusions if only a limited number of samples in a large system were analysed, this study has demonstrated the presence of important and active microbial communities, in terms of bacterial abundance, cell biomass and functional groups, in an area encompassing approximately $100 \mathrm{~km}^{2}$ of the aquifer system located in Doñana, corroborating parallel results obtained in another study conducted in a smaller area. Due to the homogeneity, not only for the groundwater but also for the lithologies, the spatial pattern of the unattached community was difficult to explain. Among the abiotic factors that usually control the spatial distribution, depth did not play any role, at least in the studied range, and grain size seemed to exert a moderate control on bacterial abundance, although less clear on cell biomass. It is, however, plausible that hydrogeology, through some other related variables such as permeability, porosity or transmissivity, plays a more important role, controlling the spatial pattern of microbial communities, although unfortunately, there are no hydrogeological models with adequate scales for groundwater microbiology studies. Moreover, nitrate seemed to play an important role in controlling bacterial abundance and biomass, because the higher the nitrate concentration, the higher the bacterial abundance, cell biomass and bacterial biomass. On the other hand, all boreholes (except wells SO1, SO2a and SO2b) sampled more than three times throughout the study period showed a clear temporal pattern, mainly during 2003, with higher bacterial abundances, cell biomasses and bacterial biomasses during summers and autumns than during springs and winters, showing the microbial communities of this aquifer system to be highly reactive. Temperature was the most important factor controlling this temporal pattern, triggering not only abundance and carbon content but also activity, mainly IRB activity. As a result, when referring to microbial ecology in the aquifer system of Doñana, there is a need to talk of a dynamic system, probably more similar to the surface aquatic systems located in the same area than to other aquifers. Finally, hydrogeological models jointly developed between microbiologists and hydrogeologists will provide an understanding of the control exerted by hydrology (rainfall, hydrogeological flows, hydrological relationships between groundwater and surface aquatic systems) on the microbial communities of this aquifer system, a phenomenon that has been partially described in this study.

\section{ACKNOWLEDGEMENTS}

This study was supported by the Spanish CICYT grant no. REN2002-10221-E/HID. Sergio Velasco was provided with a personal grant from the Spanish Department of Education and Science (FPI-2001-2175). We thank Pablo Acebes and the Doñana Biological Reserve staff for field help.

\section{REFERENCES}

Alfreider A, Krossbacher M, Psenner R (1997) Groundwater samples do not reflect bacterial densities and activity in subsurface systems. Water Research 31, 832-840.

APHA, AWWA, WPCF (1987) Standard Methods for the Examination of Water and Wastewater. American Public Health Association, Washington DC.

Baker MA, Valett HM, Dahm CN (2000) Organic carbon supply and metabolism in a shallow groundwater ecosystem. Ecology $\mathbf{8 1}$, 3133-3148.

Balkwill DL (1989) Numbers, diversity, and morphologicalcharacteristics of aerobic, chemoheterotrophic bacteria in deep subsurface sediments from a site in south-Carolina. Geomicrobiology Journal 7, 33-52.

Balkwill DL, Fredrickson JK, Thomas JM (1989) Vertical and horizontal variations in the physiological diversity of the aerobic chemoheterotrophic bacterial microflora in deep southeast coastal-plain subsurface sediments. Applied and Environmental Microbiology 55, 1058-1065.

Balkwill DL, Ghiorse WC (1985) Characterization of subsurface bacteria associated with 2 shallow aquifers in Oklahoma. Applied and Environmental Microbiology 50, 580-588.

Balkwill DL, Murphy EM, Fair DM, Ringelberg DB, White DC (1998) Microbial communities in high and low recharge environments: Implications for microbial transport in the vadose zone. Microbial Ecology 35, 156-171.

Beloin RM, Sinclair JL, Ghiorse WC (1988) Distribution and activity of microorganisms in subsurface sediments of a pristine study site in Oklahoma. Microbial Ecology 16, 85-97.

Bengtsson G (1989) Growth and metabolic flexibility in groundwater bacteria. Microbial Ecology 18, 235-248.

Bennett PC, Hiebert FK, Rogers JR (2000) Microbial control of mineral-groundwater equilibria: macroscale to microscale. Hydrogeology Journal 8, 47-62.

Bölter M, Bloem J, Meiners K, Möller R (2002) Enumeration and biovolume determination of microbial cells - a methodological review and recommendations for applications in ecological research. Biology and Fertility of Soils 36, 249-259.

Bone TL, Balkwill DL (1988) Morphological and cultural comparison of microorganisms in surface soil and subsurface sediments at a pristine study site in Oklahoma. Microbial Ecology 16, 49-64.

Bratbak G (1993) Microscope methods for measuring bacterial biovolume: epifluorescence microscopy, scanning electron microscopy and transmission electron microscopy. In Handbook of 
Methods in Aquatic Microbial Ecology (eds Kemp PF, Sherr BF, Sherr EB, Cole JJ). Lewis Publishers, Boca Raton, FL, pp. 309-317.

Brockman FJ, Murray CJ (1997) Subsurface microbiological heterogeneity: current knowledge, descriptive approaches and applications. FEMS Microbiology Reviews 20, 231-247.

Brugger A, Reitner B, Kolar I, Queric N, Herndl GJ (2001) Seasonal and spatial distribution of dissolved and particulate organic carbon and bacteria in the bank of an impounding reservoir on the Enns River, Austria. Freshwater Biology 46, 997-1016.

Chapelle FH (2000a) The significance of microbial processes in hydrogeology and geochemistry. Hydrogeology Journal 8, 41-46.

Chapelle FH (2000b) Groundwater Microbiology and Geochemistry. John Wiley \& Sons, New York, NY.

Chapelle FH, Lovley DR (1990) Rates of microbial-metabolism in deep coastal-plain aquifers. Applied and Environmental Microbiology 56, 1865-1874.

Coleto MC (2003) Funciones hidrológicas y biogeoquímicas de las formaciones palustres de los mantos eólicos de El Abalario-Doñana (Huelva). PhD Thesis, Universidad Autónoma de Madrid, Madrid, Spain.

Cullimore DR (1993) Practical Manual of Groundwater Microbiology. Lewis Publishers, Chelsea, MI.

Danielopol DL (1989) Groundwater fauna associated with riverine aquifers. Journal of the North American Benthological Society $\mathbf{8}$, $18-35$.

Danielopol DL, Griebler C, Gunatilaka A, Notenboom J (2003) Present state and future prospects for groundwater ecosystems. Environmental Conservation 30, 104-130.

Danielopol DL, Niederreiter R (1987) A sampling device for groundwater organisms and oxygen measurement in multi-level monitoring wells. Stygologia 3, 252-263.

Dassonville F, Renault P, Valles V (2004) A model describing the interactions between anaerobic microbiology and geochemistry in a soil amended with glucose and nitrate. European Journal of Soil Science 55, 29-45.

Dunlap WJ, McNabb JF, Scalf MR, Cosby RL, Kerr RS (1977) Sampling for Organic Chemicals and Microorganisms in the Subsurface. Office of Research and Development, US Environmental Protection Agency, Ada, OK.

Eydal HSC, Pedersen K (2007) Use of an ATP assay to determine viable microbial biomass in Fennoscandian Shield groundwater from depths of 3-1000 m. Journal of Microbiological Methods 70, $363-373$.

Fredrickson JK, Balkwill DL, Zachara JM, Li SMW, Brockman FJ, Simmons MA (1991) Physiological diversity and distributions of heterotrophic bacteria in deep Cretaceous sediments of the Atlantic coastal-plain. Applied and Environmental Microbiology 57, 402-411.

Fredrickson JK, McKinley JP, Bjornstad BN, Long PE, Ringelberg DB, White DC, Krumholz LR, Suflita JM, Colwell FS, Lehman RM, Phelps TJ, Onstott TC (1997) Pore-size constraints on the activity and survival of subsurface bacteria in a late Cretaceous shale-sandstone sequence, northwestern New Mexico. Geomicrobiology Journal 14, 183-202.

Fredrickson JK, Phelps TJ (1997) Subsurface drilling and sampling. In Manual of Environmental Microbiology (eds Hurst CJ, Knudsen GR, McInerney MJ, Stetzenbach LD, Walter MV). American Society of Microbiology, Washington DC, pp. 526-540.

Fredrickson JK, Zachara JM, Balkwill DL, Kennedy D, Li SMW, Kostandarithes HM, Daly MJ, Romine MF, Brockman FJ (2004) Geomicrobiology of high-level nuclear waste-contaminated vadose sediments at the Hanford Site, Washington State. Applied and Environmental Microbiology 70, 4230-4241.
Fry JC (1990) Direct methods and biomass estimation. Methods in Microbiology 22, 41-85.

Goldscheider N, Hunkeler D, Rossi P (2006) Review: Microbial biocenoses in pristine aquifers and an assessment of investigative methods. Hydrogeology Journal 14, 926-941.

Gounot AM (1996) Microbial ecology of groundwaters. In Algal Ecology: Freshwater Benthic Ecosystems (eds Stevenson RJ, Bothwell MI, Lowe RI). Academic Press, San Diego, CA, pp. 189-215.

Griebler C, Mindl B, Slezak D, Geiger-Kaiser M (2002) Distribution patterns of attached and suspended bacteria in pristine and contaminated shallow aquifers studied with an in situ sediment exposure microcosm. Aquatic Microbial Ecology 28, 117-129.

Haack SK, Fogarty LR, West TG, Alm EW, McGuire JT, Long DT, Hyndman DW, Forney LJ (2004) Spatial and temporal changes in microbial community structure associated with recharge-influenced chemical gradients in a contaminated aquifer. Environmental Microbiology 6, 438-448.

Hancock PJ, Boulton AJ, Humphreys WF (2005) Aquifers and hyporheic zones: towards an ecological understanding of groundwater. Hydrogeology Journal 13, 98-111.

Harvey RW, George LH (1987) Growth determinations for unattached bacteria in a contaminated aquifer. Applied and Environmental Microbiology 53, 2992-2996.

Harvey RW, Smith RL, George L (1984) Effect of organic contamination upon microbial distributions and heterotrophic uptake in a Cape-Cod, Mass., aquifer. Applied and Environmental Microbiology 48, 1197-1202.

Hazen TC, Jiménez L, Devictoria GL, Fliermans CB (1991) Comparison of bacteria from deep subsurface sediment and adjacent groundwater. Microbial Ecology 22, 293-304.

Hirsch P, Rades-Rohkohl E (1990) Microbial colonization of aquifer sediment exposed in a groundwater well in northern Germany. Applied and Environmental Microbiology 56, 2963-2966.

Kieft TL, Murphy EM, Haldeman DL, Amy PS, Bjornstad BN, McDonald EV, Ringelberg DB, White DC, Stair J, Griffiths RP, Gsell TC, Holben WE, Boone DR (1998) Microbial transport, survival, and succession in a sequence of buried sediments. Microbial Ecology 36, 336-348.

Kirchman D, Sigda J, Kapuscinski R, Mitchell R (1982) Statistical-analysis of the direct count method for enumerating bacteria. Applied and Environmental Microbiology 44, 376-382.

Kölbel-Boelke J, Anders EM, Nehrkorn A (1988) Microbial communities in the saturated groundwater environment. 2 Diversity of bacterial communities in a Pleistocene sand aquifer and their in vitro activities. Microbial Ecology 16, 31-48.

Legendre P, Legendre L (1998) Numerical Ecology. Elsevier, Amsterdam, The Netherlands.

Lehman RM, Colwell FS, Bala GA (2001) Attached and unattached microbial communities in a simulated basalt aquifer under fracture- and porous-flow conditions. Applied and Environmental Microbiology 67, 2799-2809.

Lehman RM, O'Connell SP, Banta A, Fredrickson JK, Reysenbach AL, Kieft TL, Colwell FS (2004) Microbiological comparison of core and groundwater samples collected from a fractured basalt aquifer with that of dialysis chambers incubated in situ. Geomicrobiology Journal 21, 169-182.

López-Archilla AI, Moreira D, Velasco S, López-Garcia P (2007) Archaeal and bacterial community composition of a pristine coastal aquifer in Doñana National Park, Spain. Aquatic Microbial Ecology 47, 123-139.

Madsen EL, Ghiorse WC (1993) Groundwater microbiology: subsurface ecosystem processes. In Aquatic Microbiology: An Ecological Approach (ed. Ford TE). Blackwell Scientific Publications, Cambridge, MA, pp. 167-213.

Manzano M, Custodio E (2007) Groundwater baseline chemistry in 
the Doñana aquifer (SW Spain) and geochemical controls. In Baseline Groundwater Quality (eds Edmunds M, Shand P). Blackwell, London, UK, pp. 23-35.

Manzano M, Custodio E, Lozano E, Higueras H (2007) Relationships between wetlands and the Doñana coastal aquifer (SW Spain). In Proceedings of the XXXV IAH Congress (eds Ribeiro L, Chambel A, Condesso de Melo MT), Lisbon, Portugal, pp. 1-10. Martino DP, Grossman EL, Ulrich GA, Burger KC, Schlichenmeyer JL, Suflita JM, Ammerman JW (1998) Microbial abundance and activity in a low-conductivity aquifer system in east-central Texas. Microbial Ecology 35, 224-234.

Marxsen J (1988) Investigations into the number of respiring bacteria in groundwater from sandy and gravelly deposits. Microbial Ecology $16,65-72$.

Mauck BS, Roberts JA (2007) Mineralogic control on abundance and diversity of surface-adherent microbial communities. Geomicrobiology Journal 24, 167-177.

McLean JE, Dupont RR, Sorensen DL (2006) Iron and arsenic release from aquifer solids in response to biostimulation. Journal of Environmental Quality 35, 1193-1203.

Mehnert E, Hwang HH, Johnson TM, Sanford RA, Beaumont WC, Holm TR (2007) Denitrification in the shallow ground water of a tile-drained, agricultural watershed. Journal of Environmental Quality 36, 80-90.

Mikell AT, Smith CL, Richardson JC (1996) Evaluation of media and techniques to enumerate heterotrophic microbes from karst and sand aquifer springs. Microbial Ecology 31, 115-124.

Montes C, Borja F, Bravo MA, Moreira JM (1998) Doñana: Una Aproximación Ecosistémica. Consejería de Medio Ambiente, Junta de Andalucía, Sevilla, Spain.

Murphy EM, Schramke JA (1998) Estimation of microbial respiration rates in groundwater by geochemical modeling constrained with stable isotopes. Geochimica et Cosmochimica Acta 62, 3395-3406.

Musslewhite CL, McInerney MJ, Dong HL, Onstott TC, Green-Blum M, Swift D, Macnaughton S, White DC, Murray C, Chien YJ (2003) The factors controlling microbial distribution and activity in the shallow subsurface. Geomicrobiology Journal 20, 245-261.

Pedersen K, Arlinger J, Hallbeck L, Pettersson C (1996) Diversity and distribution of subterranean bacteria in groundwater at Oklo in Gabon, Africa, as determined by $16 \mathrm{~S}$ rRNA gene sequencing. Molecular Ecology 5, 427-436.

Pedersen K, Ekendahl S (1990) Distribution and Activity of Bacteria in deep granitic groundwaters of southeastern Sweden. Microbial Ecology 20, 37-52.

Penny E, Lee MK, Morton C (2003) Groundwater and microbial processes of Alabama coastal plain aquifers. Water Resources Research 39, XX-XX.

Phelps TJ, Pfiffner SM, Sargent KA, White DC (1994) Factors influencing the abundance and metabolic capacities of microorganisms in eastern coastal-plain sediments. Microbial Ecology 28, 351-364.

Psenner R (1993) Determination of size and morphology of aquatic bacteria by automated image analysis. In Handbook of Methods in Aquatic Microbial Ecology (eds Kemp PF, Sherr BF, Sherr EB, Cole JJ). Lewis Publishers, Boca Raton, FL, pp. 339-345.

Quinn GP, Keough MJ (2002) Experimental Design and Data Analysis for Biologists. Cambridge University Press, Cambridge, UK.

Roadcap GS, Sanford RA, Jin QS, Pardinas JR, Bethke CM (2006) Extremely alkaline $(\mathrm{pH}>12)$ ground water hosts diverse microbial community. Ground Water 44, 511-517.

Santoro AE, Boehm AB, Francis CA (2006) Denitrifier community composition along a nitrate and salinity gradient in a coastal aquifer. Applied and Environmental Microbiology 72, 2102-2109.

Shi Y, Zwolinski MD, Schreiber ME, Bahr JM, Sewell GW, Hickey WJ (1999) Molecular analysis of microbial community structures in pristine and contaminated aquifers: field and laboratory microcosm experiments. Applied and Environmental Microbiology 65, 2143-2150.

Sinclair JL, Ghiorse WC (1989) Distribution of aerobic-bacteria, protozoa, algae, and fungi in deep subsurface sediments. Geomicrobiology Journal 7, 15-31.

Trick T, Custodio E (2004) Hydrodynamic characteristics of the western Doñana Region (area of El Abalario), Huelva, Spain. Hydrogeology Journal 12, 321-335.

Trojan MD, Maloney JS, Stockinger JM, Eid EP, Lahtinen MJ (2003) Effects of land use on ground water quality in the Anoka sand plain aquifer of Minnesota. Ground Water 41, 482-492.

Velasco S, Acebes P, López-Archilla AI, Montes C, Guerrero MC (2008) Environmental factors controlling the spatiotemporal distribution of microbjal communities in a coastal, sandy aquifer system (Doñana, $\bar{\equiv}$ west Spain). Hydrogeology Journal XX, $\mathrm{XXX}-\mathrm{XX}$. in press.

Viollier E, Inglett PW, Hunter K, Roychoudhury AN, Van Cappellen $\mathrm{P}$ (2000) The ferrozine method revisited: $\mathrm{Fe}$ (II)/Fe (III) determination in natural waters. Applied Geochemistry 15, $785-790$

Vorobyova E, Soina V, Gorlenko M, Minkovskaya N, Zalinova N, Mamukelashvili A, Gilichinsky D, Rivkina E, Vishnivetskaya T (1997) The deep cold biosphere: facts and hypothesis. FEMS Microbiology Reviews 20, 277-290.

Whitman WB, Coleman DC, Wiebe WJ (1998) Prokaryotes: The unseen majority. Proceedings of the National Academy of Sciences of the United States of America 95, 6578-6583.

Zar JH (1998) Biostatistical Analysis. Prentice Hall, New York, NY. Zhou JZ, Xia BC, Huang H, Palumbo AV, Tiedje JM (2004) Microbial diversity and heterogeneity in sandy subsurface soils. Applied and Environmental Microbiology 70, 1723-1734. 


\section{Author Query Form}

\section{Journal: Geobiology}

\section{Article: gbi_183.fm}

Dear Author,

During the copy-editing of your paper, the following queries arose. Please respond to these by marking up your proofs with the necessary changes/additions. Please write your answers on the query sheet if there is insufficient space on the page proofs. Please write clearly and follow the conventions shown on the attached corrections sheet. If returning the proof by fax do not write too close to the paper's edge. Please remember that illegible mark-ups may delay publication.

Many thanks for your assistance.

\begin{tabular}{|l|l|l|}
\hline No. & Query & Remarks \\
\hline $\mathbf{1}$ & Author: By 'bact $\mathrm{mL}^{-1}{ }^{\prime}$, do you mean 'number of bacteria per mL'? & \\
\hline $\mathbf{2}$ & $\begin{array}{l}\text { Blackwell-Wiley: Please supply dates this manuscript was received and } \\
\text { accepted for publication. }\end{array}$ & \\
\hline $\mathbf{3}$ & Author: Please supply telephone and fax numbers of the corresponding author. & \\
\hline $\mathbf{4}$ & Author: Please confirm insertion of 'with' after 'conform'. & \\
\hline $\mathbf{5}$ & Author: Please check this symbol 'Ø'. & \\
\hline $\mathbf{6}$ & Author: Please confirm manufacturer's details inserted here for Millipore filter. & \\
\hline $\mathbf{7}$ & $\begin{array}{l}\text { Author: Please supply manufacturer's details for Olympus IX50 inverted } \\
\text { microscope. }\end{array}$ & $\begin{array}{l}\text { Author: Trick 1998 has not been found in the reference list. Please supply full } \\
\text { bibliographic details. }\end{array}$ \\
\hline $\mathbf{8}$ & $\begin{array}{l}\text { Author: Please check this sentence 'Tukey's HSD test was employed, as an a } \\
\text { posteriori method, to compare pairs of means after ANOVA testing' as it seems a } \\
\text { repetition of the above sentence. }\end{array}$ & \\
\hline $\mathbf{9}$ & Author: Please confirm manufacturer's inserted here for Statistica 6.0. & \\
\hline $\mathbf{1 1}$ & Author: Please supply page range for Penny et al. 2003. & \\
\hline $\mathbf{1 2}$ & Author: Any update on the publication of Velasco et al. in press. & \\
\hline
\end{tabular}




\section{Please correct and return this set}

Please use the proof correction marks shown below for all alterations and corrections. If you wish to return your proof by fax you should ensure that all amendments are written clearly in dark ink and are made well within the page margins.

\begin{tabular}{|c|c|c|}
\hline Instruction to printer & Textual mark & Marginal mark \\
\hline Leave unchanged & ... under matter to remain & ( ) \\
\hline $\begin{array}{l}\text { Insert in text the matter } \\
\text { indicated in the margin }\end{array}$ & $h$ & $\begin{array}{l}\text { New matter followed by } \\
h \text { or } h \otimes\end{array}$ \\
\hline Delete & $\begin{array}{l}\text { I through single character, rule or underline } \\
\text { or }\end{array}$ & $\sigma$ or $\sigma / \otimes$ \\
\hline $\begin{array}{l}\text { Substitute character or } \\
\text { substitute part of one or } \\
\text { more word(s) }\end{array}$ & / through letter or & $\begin{array}{l}\text { new character / or } \\
\text { new characters / }\end{array}$ \\
\hline Change to italics & — under matter to be changed & $\leftarrow$ \\
\hline Change to capitals & $\equiv$ under matter to be changed & $\equiv$ \\
\hline Change to small capitals & $=$ under matter to be changed & $=$ \\
\hline Change to bold type & $\sim$ under matter to be changed & $\sim$ \\
\hline Change to bold italic & $\approx$ under matter to be changed & $\underline{s i n}$ \\
\hline Change to lower case & Encircle matter to be changed & $\not$ \\
\hline Change italic to upright type & (As above) & \\
\hline Change bold to non-bold type & (As above) & \\
\hline Insert 'superior' character & $\begin{array}{l}\text { I through character or } \\
K \text { where required }\end{array}$ & $\begin{array}{l}y^{\prime} \text { or } y \\
\text { under character } \\
\text { e.g. } y^{2} \text { or } y^{2}\end{array}$ \\
\hline Insert 'inferior' character & (As above) & $\begin{array}{l}\text { L } \\
\text { over character } \\
\text { e.g. } \hat{\imath}\end{array}$ \\
\hline Insert full stop & (As above) & $\odot$ \\
\hline Insert comma & (As above) & , \\
\hline Insert single quotation marks & (As above) & $\begin{array}{l}\dot{y} \text { or } \dot{X} \text { and/or } \\
\dot{y} \text { or } \dot{X}\end{array}$ \\
\hline Insert double quotation marks & (As above) & $\begin{array}{l}\dddot{y} \text { or } \ddot{x} \text { and/or } \\
\ddot{y} \text { or } \ddot{x}\end{array}$ \\
\hline Insert hyphen & (As above) & $1-1$ \\
\hline Start new paragraph & $\digamma$ & 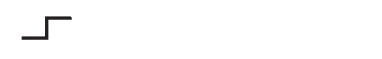 \\
\hline No new paragraph & $\infty$ & $\omega$ \\
\hline Transpose & $\sqcup$ & ᄃ \\
\hline Close up & linking $\bigcirc$ characters & \\
\hline $\begin{array}{l}\text { Insert or substitute space } \\
\text { between characters or words }\end{array}$ & $\begin{array}{l}\text { I through character or } \\
\Lambda \text { where required }\end{array}$ & \\
\hline $\begin{array}{l}\text { Reduce space between } \\
\text { characters or words }\end{array}$ & $\begin{array}{l}\text { between characters or } \\
\text { words affected }\end{array}$ & $\uparrow$ \\
\hline
\end{tabular}

